\title{
Time-dependent Pancharatnam phases and quantum correlations for coupling superconducting two-qubit system with dissipative environment
}

\author{
LIYUAN XUE ${ }^{1}$, Z.S. WANG ${ }^{2}$ \\ ${ }^{1}$ Quantum Information Research Center, Shangrao Normal University, \\ Shangrao 334001, P.R. China \\ ${ }^{2}$ College of Physics and Communication Electronics, Jiangxi Normal University, \\ Nanchang 330022, P.R. China
}

\begin{abstract}
Two coupling superconducting qubits are studied for the quantum concurrence, discord, and Pancharatnam phase, for the $\mathrm{X}$ and $\mathrm{Y}$ states under the dephasing and instantaneous decay environment as well as their couplings. We find that the $\mathrm{X}$ and $\mathrm{Y}$ states are special mixed states according to the Bloch radius. In general, the larger the environment and phonon number are at the larger region of time, the larger the quantum concurrence and discord are. But we find that the environment correlations are helpful to implement the quantum computation. The Pancharatnam phases provide a way to distinguish the $\mathrm{X}$ and $\mathrm{Y}$ states.
\end{abstract}

Keywords: Pancharatnam phases, quantum concurrence, quantum discord, dissipative environment, Bloch radius.

\section{Introduction}

By using quantum nonlocality, i.e., quantum entanglement $[\underline{1}, \underline{2}]$, quantum discord $[\underline{3}, \underline{4}]$ and geometric phase [ $\underline{5}-\underline{7}$ ], quantum computing is far more efficient than its classical counterparts in factoring large numbers, searching databases and simulating physical systems $[\underline{8}, \underline{9}]$. All the aspects of quantum computing are currently under intensive study. One of the key challenges from quantum computing and information is to overcome the decoherent effects of physical system in order to preserve the quantum correlations in the real situation.

Another crucial requirement in implementing quantum logic devices is needed to have a high degree of quantum coherence [10-12]. But the unavoidable interactions between the physical system and its environment makes the quantum coherence be extremely fragile and susceptible to decay away. If the coherence in the quantum qubits system [13] is lost, the qubits become unentangled. Such a decoherent process limits 
the ability of quantum information $[\underline{8}, \underline{14}]$. Therefore, it is a practical requirement to consider the decoherent effect on the quantum system and its robustness against the decoherence [15].

It is difficult to predict the coherence time [16] of the qubits system because all possible couplings to the other quantum degrees of freedom must be considered. Thus, it is necessary to study the dynamic evolution of dissipative system by turning off the interaction between the qubit and its environment, which can efficiently preserve the qubit coherence and correlation. Unfortunately, this generally decouples the corrections among the qubits so as not to prevent two-qubit gate operations [17]. Especially, the decoupling and the quantum gate are separated in time; such an unprotected gate is very susceptible to decoherence induced errors.

It is known that the geometric phase embedded in the geometric structure [18] depends only on the symplectic area spanned in the evolving path of quantum system. Thus the geometric quantum computation is intrinsically fault-tolerant scheme and therefore resistent to fluctuating errors from the quantum system. The geometric phase including the decoherence effects has attracted increasing interests in both fundamental concepts and wide applications. Therefore, it is important to understand the geometric phase and implement geometric quantum computation in real physical systems [19-22].

On the other hand, the solid-state quantum gate from the superconducting qubits [23-26] may be a promising candidate for a quantum computer. In the experiments, two kinds of elementary linear logic gates [27-30] are sufficient in processing or implementing an arbitrary operation on qubits in order to achieve universal quantum computation. For the geometric quantum computation, therefore, it is important to study the geometric phases [31, 32] and quantum correlations [33, 34] of superconducting two-qubit system.

In this paper, we investigate firstly the time-dependent geometric phase of two coupling superconducting qubits system with dissipative effects under the situation of the initially entangled superconducting qubits state. Then we discuss the relations between the geometric phases and quantum correlations. By comparing the time-dependent geometric phases and quantum correlations in both the $\mathrm{X}$ and the $\mathrm{Y}$ states, at last, we find that the time-dependent Pancharatnam phases reflect the message of quantum correlations beside keeping the motion memory of such a coupling superconducting twoqubit system.

\section{Coupling superconducting two-qubit in open system}

In a quantum system, the basic memory units are a set of the quantum logic gates with a number of qubits. The quantum gates of a pure quantum state transform an initial state into a state in terms of unitary matrices and therefore quantum logic gates are reversible according to the unitary property. The most common quantum gates operation can be simulated by two coupling qubits. For quantum open system, however, it is necessary to study the dynamic behavior of two-qubit system. 


\section{Environment}

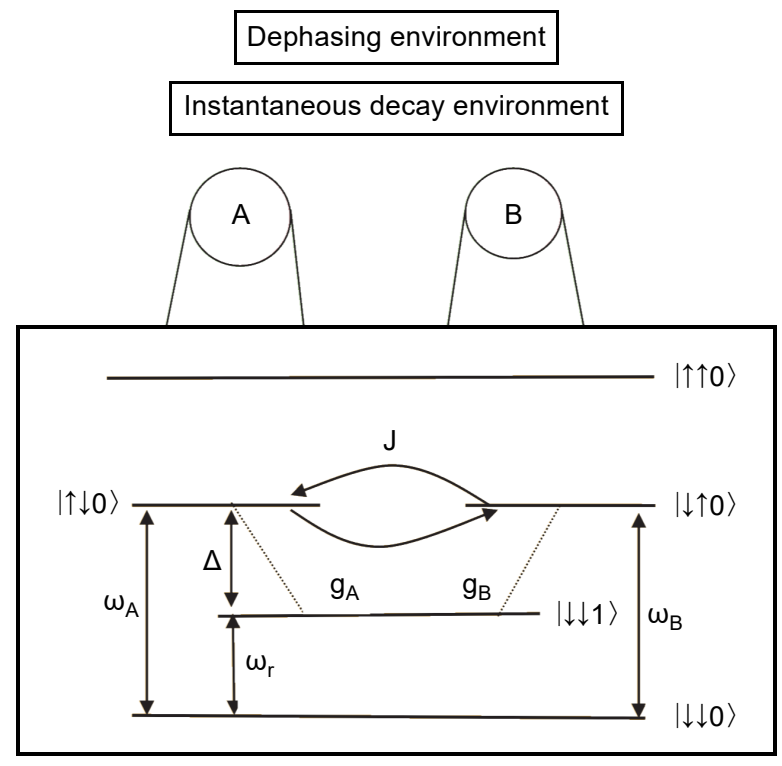

Fig. 1. The qubits A and B independently interact with respective environments.

Thus, let us start from two coupling superconducting qubits, shown schematically in Fig. 1, where the resonator plus qubit Hamiltonian is taken as a Jaynes-Cummings form [35],

$$
H_{\mathrm{JC}}=\hbar \omega_{\mathrm{r}} a^{\dagger} a+\sum_{j=\mathrm{A}, \mathrm{B}} \hbar \frac{\omega_{\mathrm{a}, j}}{2} \sigma_{j}^{z}-\sum_{j=\mathrm{A}, \mathrm{B}} \hbar g_{j}\left(a^{\dagger} \sigma_{j}^{-}+\sigma_{j}^{+} a\right)
$$

where $\omega_{\mathrm{r}}$ is a resonance frequency, $\omega_{\mathrm{a}, j}$ is the transition frequency of qubit $j, g_{j}$ is the coupling strength of the resonator to qubit $j, \sigma_{j}^{z}$ is Pauli matrices of qubit $j, \sigma_{j}^{ \pm}$is raising and lowering operators.

To avoid excitation of the resonator, we assume that both qubits are strongly detuned from the resonator $\left|\Delta_{i}\right|=\left|\omega_{\mathrm{a}, j}-\omega_{\mathrm{r}}\right| \gg g_{j}$. In this situation, we adiabatically eliminate the resonant Jaynes-Cummings interaction by

$$
U=\exp \left(\frac{g_{\mathrm{A}}}{\Delta_{\mathrm{A}}}\left(a^{\dagger} \sigma_{\mathrm{A}}^{-}-a \sigma_{\mathrm{A}}^{+}\right)+\frac{g_{\mathrm{B}}}{\Delta_{\mathrm{B}}}\left(a^{\dagger} \sigma_{\mathrm{B}}^{-}-a \sigma_{\mathrm{B}}^{+}\right)\right)
$$

and the full system with the two qubits and the cavity is described by the effective Hamiltonian [푸,$\underline{37}]$,

$$
\mathcal{H}=\frac{\hbar \omega_{\mathrm{A}}}{2} \sigma_{\mathrm{A}}^{z}+\frac{\hbar \omega_{\mathrm{B}}}{2} \sigma_{\mathrm{B}}^{z}+\hbar\left(\omega_{\mathrm{r}}+\chi_{\mathrm{r}, \mathrm{A}} \sigma_{\mathrm{A}}^{z}+\chi_{\mathrm{r}, \mathrm{B}} \sigma_{\mathrm{B}}^{z}\right) a^{\dagger} a+\hbar J\left(\sigma_{\mathrm{A}}^{-} \sigma_{\mathrm{B}}^{+}+\sigma_{\mathrm{B}}^{-} \sigma_{\mathrm{A}}^{+}\right)
$$


where $\chi_{\mathrm{r}, j}=g_{j}^{2} / \Delta_{j}(j=\mathrm{A}, \mathrm{B})$ is a coupling strength between the resonator and the qubits $j$ and $\omega_{j}=\omega_{\mathrm{a}, j}+\chi_{\mathrm{r}, j}(j=\mathrm{A}, \mathrm{B})$ are a transition frequency, and

$$
J=\frac{g_{\mathrm{A}} g_{\mathrm{B}}\left(\Delta_{\mathrm{A}}+\Delta_{\mathrm{B}}\right)}{2 \Delta_{\mathrm{A}} \Delta_{\mathrm{B}}}
$$

is an interacting strength between the two qubits and the cavity $a^{\dagger} a=n$.

The interaction between the two-qubit system and its environment will be given by a superoperator $\mathcal{L}$. The time evolution of quantum open system is generated by a Lindbladian-type master equation [38],

$$
\dot{\rho}=-\frac{i}{\hbar}[\mathcal{H}, \rho]+\mathcal{L} \rho
$$

under the condition of weak coupling inside the system, the Lindbladian-type master equation is relatively accurate. But if you have a strong coupling inside the system, it is not very accurate, because after all, this equation is derived from a lot of approximations.

If all details of the system-environment iteration are known, in principle, one can construct the corresponding superoperator $\mathcal{L}$. In most situations, however, the decoherent ways of open system are very complicated. Therefore, it is interesting to consider the mixture of both the dephasing and instantaneous two-decay mode. In this case, $\mathcal{L} \rho$ may be expressed as

$$
\begin{aligned}
\mathcal{L} \rho= & -\sum_{i=\mathrm{A}, \mathrm{B}} \Gamma_{i}\left(S_{i}^{z} S_{i}^{z} \rho-2 S_{i}^{z} \rho S_{i}^{z}+\rho S_{i}^{z} S_{i}^{z}\right) \\
& -2 \Gamma_{0}\left(S_{\mathrm{A}}^{z} S_{\mathrm{B}}^{z} \rho-S_{\mathrm{B}}^{z} \rho S_{\mathrm{A}}^{z}+\rho S_{\mathrm{A}}^{z} S_{\mathrm{B}}^{z}-S_{\mathrm{A}}^{z} \rho S_{\mathrm{B}}^{z}\right) \\
& -\sum_{j, k=\mathrm{A}, \mathrm{B}} \frac{\Gamma_{j k}}{2}\left(S_{j}^{+} S_{k}^{-} \rho-2 S_{k}^{-} \rho S_{j}^{+}+\rho S_{j}^{+} S_{k}^{-}\right)
\end{aligned}
$$

where $S_{\mathrm{A}}^{z}=\sigma_{\mathrm{A}}^{z} / 2$ and $S_{\mathrm{B}}^{z}=\sigma_{\mathrm{B}}^{z} / 2$ are spin operators for the qubits A and $\mathrm{B}, S^{+}=a \sigma_{+}$ is a product of the annihilation operator $a$ and raising $\sigma_{+}, S^{-}=a^{\dagger} \sigma_{-}$is a product of the creation operator and lowering $\sigma_{-}, \Gamma_{i}$ are the dephasing rates, $\Gamma_{0}$ is a correlated dephasing rate which describes the correlation between the environments of two qubits as well as $\Gamma_{j k}$ describes the correlation between the environments of two qubits under the instantaneous decay mode.

Generally, $\Gamma_{i}$ and $\Gamma_{j k}$ are dependent on each other under the case of non-correlated environments, thus we can take the autocorrelated environments for the two-qubit system in the following, i.e., $\Gamma_{j k}=\Gamma$ and $\Gamma_{j j}=\gamma$, which facilitate the investigation into the relation between the dephasing and instantaneous decays, and the ratios between them affect the geometric phase and quantum correlation. 


\section{Bloch sphere structure and geometric phase of two-qubit system}

A mixed state density matrix $\rho(t)$ can be expressed as

$$
\rho(t)=\sum_{k=1}^{N} \alpha_{k}^{2}(t)\left|\psi_{k}(t)\right\rangle\left\langle\psi_{k}(t)\right|
$$

where $\alpha_{k}(t)$ are a set of classical probability amplitudes with the normalized condition,

$$
\sum_{k=1}^{N} \alpha_{k}^{2}(t)=1
$$

For the geometric phase of a mixed state, a usual way is to permit a nonunitary evolution with the nonunit state vectors $\left|\Psi_{k}(t)\right\rangle$ in the complex Hilbert space $H_{N}=\left|\Psi_{k}(t)\right\rangle$ $=\alpha_{k}\left|\psi_{k}(t)\right\rangle ; k=1,2, \ldots, N$. It is known that the invariant proposed by Bargmann is an approach to distinguish unitary evolution from nonunitary one. In order to preserve the unitary property in the study of the mixed state, especially for keeping $\bigotimes_{k=1}^{4} U(1)$ gauge invariants in both the density matrix and geometric phase, we construct a superspinorial representation of the mixed state vector in connection with the given density matrix, i.e. [19],

$$
|\Psi(t)\rangle=\left(\left|\Psi_{1}(t)\right\rangle,\left|\Psi_{2}(t)\right\rangle,\left|\Psi_{3}(t)\right\rangle,\left|\Psi_{4}(t)\right\rangle\right)
$$

The geometric phase of the mixed state can be obtained by the normalized spinorial representation in which the smooth curve $\zeta=|\Psi(t)\rangle$ is subdivided into $L$ parts. The points of subdivision are at $t_{i}=t_{0}+i \Delta t$ with values, where $i=1,2, \ldots, L$.

Each trajectory, then, is represented by a discrete sequence of quantum states,

$$
\left(\left|\Psi\left(t_{0}\right)\right\rangle,\left|\Psi\left(t_{1}\right)\right\rangle, \ldots,\left|\Psi\left(t_{L}\right)\right\rangle,\left|\Psi\left(t_{L+1}\right)\right\rangle\right)=|\Psi(t)\rangle
$$

and each $\left\langle\Psi\left(t_{i}\right) \mid \Psi\left(t_{i+1}\right)\right\rangle$ is corresponding to a phase. By using the products of $L$ short geodesic arcs connecting $\left|\Psi\left(t_{i}\right)\right\rangle$ with $\left|\Psi\left(t_{i+1}\right)\right\rangle$, we get the Pancharatnam phase of mixed state as

$$
\begin{aligned}
\gamma_{\mathrm{P}}(t) & =-\lim _{L \rightarrow \infty}\left\langle\Psi\left(t_{0}\right) \mid \Psi\left(t_{1}\right)\right\rangle\left\langle\Psi\left(t_{1}\right) \mid \Psi\left(t_{2}\right)\right\rangle \ldots\left\langle\Psi\left(t_{L}\right) \mid \Psi\left(t_{L+1}\right)\right\rangle\left\langle\Psi\left(t_{L+1}\right) \mid \Psi\left(t_{0}\right)\right\rangle \\
= & \arg \left\langle\Psi\left(t_{0}\right) \mid \Psi(t)\right\rangle-\lim _{L \rightarrow \infty} \arg \prod_{i=0}^{L}\left\langle\Psi\left(t_{i}\right) \mid \Psi\left(t_{i+1}\right)\right\rangle
\end{aligned}
$$

Using the normalized condition and the limiting process, we obtain

$$
\gamma_{\mathrm{P}}(t)=\sum_{k=1}^{4} \arg \left\langle\Psi_{k}\left(t_{0}\right) \mid \Psi_{k}(t)\right\rangle-\mathfrak{I} \sum_{k=1}^{4} \int_{t_{0}}^{t}\left\langle\Psi_{k}(t)|\mathrm{d}| \Psi_{k}(t)\right\rangle
$$


which means that $\gamma_{\mathrm{P}}(t)$ in Eq. (12) is a physical quantity with the gauge invariant. In order to express exactly the geometric properties, we can map the coupling superconducting two-qubit system onto a general Bloch sphere structure.

The density matrix can be expanded in terms of a set of traceless and Hermitian matrices. Under the situation of two-qubit system, the Hermitian matrices $\lambda_{i}, i=1,2, \ldots, 15$ are SU(4) elements as shown in Appendix I. Thus the density matrix is written as

$$
\rho(t)=\frac{1}{4}\left(I+b \sum_{i=1}^{15} n_{i}(t) \lambda_{i}\right)
$$

where $b=\sqrt{\frac{4 \times(4-1)}{2}}=\sqrt{6}$ is an expansive coefficient, $\mathbf{n}$ is called the generalized Bloch vector, $I$ is an $4 \times 4$ identity matrix, and $\lambda_{i}$ with the orthogonal and normalized conditions,

$$
\operatorname{Tr}\left(\lambda_{i} \lambda_{j}\right)=2 \delta_{i j}, \quad \operatorname{Tr} \lambda_{i}=0, \quad\left[\lambda_{i}, \lambda_{j}\right]=2 i f_{i j k} \lambda_{k}
$$

where $f_{i j k}=\operatorname{Tr}\left(\left[\lambda_{i}, \lambda_{j}\right] \lambda_{k}\right)$ are the $\mathrm{SU}(4)$ structure constants.

Multiplying $\lambda_{i}$ on the two sides of Eq. (13) and then using Eq. (14), we find that each component of the Bloch vector is given by

$$
n_{i}(t)=\frac{2}{\sqrt{6}} \operatorname{Tr}\left(\lambda_{i} \rho(t)\right)
$$

Obviously, the set

$$
S=\left\{\mathbf{n}(t) \in \mathbb{R}^{15}, \mathbf{n}(t) \cdot \mathbf{n}(t)=r^{2}(t), \mathbf{n}^{*}(t)=\mathbf{n}(t)\right\}
$$

is an analogue of the generalized 15-dimensional Bloch sphere, and $r$ is called the Bloch radius. Thus $\mathbf{n}^{\mathrm{R}}(t)=\mathbf{n}(t) / r$ is a unit vector on the unit sphere and can be parameterized by the 14 azimuthal angles of the Bloch sphere.

Next, we represent the density matrix in terms of a set of completely normalized and orthogonal eigenvectors $\left|\chi_{k}(t)\right\rangle$ of $\mathbf{n}^{\mathrm{R}}(t) \cdot \boldsymbol{\lambda}$ with eigenequations,

$$
\mathbf{n}^{\mathrm{R}}(t) \cdot \lambda\left|\chi_{k}(t)\right\rangle=\chi_{k}^{\prime}\left|\chi_{k}(t)\right\rangle
$$

where $\chi_{k}^{\prime}(k=1,2,3,4)$ are corresponding eigenvalues, and the eigenvectors $\left|\chi_{k}(t)\right\rangle$ satisfy the complete condition, i.e.,

$$
\sum_{k=1}^{4}\left|\chi_{k}(t)\right\rangle\left\langle\chi_{k}(t)\right|=I
$$

Using Eq. (17), we have

$$
\mathbf{n}^{\mathrm{R}}(t) \cdot \boldsymbol{\lambda}=\sum_{k=1}^{4} \chi_{k}^{\prime}\left|\chi_{k}(t)\right\rangle\left\langle\chi_{k}(t)\right|
$$


Inserting Eqs. (13) and (15) into Eq. (19), the density matrix is rewritten as

$$
\rho(t)=\sum_{k=1}^{4} \chi_{k}(t)\left|\chi_{k}(t)\right\rangle\left\langle\chi_{k}(t)\right|
$$

where $\chi_{k}=\frac{1}{4}\left(1+\sqrt{6} r(t) \chi_{k}^{\prime}\right)$ are classical probabilities of mixture in the open system. Thus the density matrix of the two-qubit system can be naturally expressed in terms of the geometric quantities, i.e., the generalized Bloch radius and azimuthal angles. From Eq. (13), we find

$$
\rho(t)\left|\chi_{k}(t)\right\rangle=\frac{1}{4}\left(1+\sqrt{6} r(t) \chi_{k}^{\prime}\right)\left|\chi_{k}(t)\right\rangle
$$

which means that the $\left|\chi_{k}(t)\right\rangle$ are also the eigenstates of the two-qubit density matrix $\rho(t)$ with the eigenvalues $\chi_{k}$.

According to Eqs. (9) and (21), the corresponding superspinorial representation of $\mathrm{N}$-level mixed state is given by

$$
|\Psi(t)\rangle=\left(\begin{array}{c}
\frac{1+\sqrt{6} r(t) \chi_{1}^{\prime}}{4}\left|\chi_{1}(t)\right\rangle \\
\frac{1+\sqrt{6} r(t) \chi_{2}^{\prime}}{4}\left|\chi_{2}(t)\right\rangle \\
\frac{1+\sqrt{6} r(t) \chi_{3}^{\prime}}{4}\left|\chi_{3}(t)\right\rangle \\
\frac{1+\sqrt{6} r(t) \chi_{4}^{\prime}}{4}\left|\chi_{4}(t)\right\rangle
\end{array}\right)^{\mathrm{T}}
$$

In terms of the spinorial representation of the density matrix, the Pancharatnam phase of the mixed state can be expressed as

$$
\gamma_{\mathrm{P}}(t)=\arg \sum_{k=1}^{4} \sqrt{\chi_{k}\left(t_{0}\right) \chi_{k}(t)}\left\langle\chi_{k}\left(t_{0}\right) \mid \chi_{k}(t)\right\rangle-\mathfrak{J} \sum_{k=1}^{4} \int_{t_{0}}^{t} \chi_{k}(t)\left\langle\chi_{k}(t)|\mathrm{d}| \chi_{k}(t)\right\rangle
$$

where $t_{0}$ is the initial time, $\chi_{k}(t)$ is the eigenvalue of the density matrix, $\left|\chi_{k}(t)\right\rangle$ is the eigenstate of the density matrix.

When the initial state of the two-qubit system is an entangled state, it is helpful to realize quantum computation in processing of quantum information. Therefore, we only consider here the $\mathrm{X}$ and $\mathrm{Y}$ states $[\underline{39}, \underline{40}]$, named from the visual appearance of the density matrix, which includes maximally entangled Bell states and Werner states. An important feature of the $\mathrm{X}$ state is that the density matrix would preserve its form 
of initial density matrix in process of time evolution. The time-dependent density matrix of the two-qubit system is given by

$$
\rho_{\mathrm{X}}(t)=\left(\begin{array}{cccc}
\rho_{11}(t) & 0 & 0 & \rho_{14}(t) \\
0 & \rho_{22}(t) & \rho_{23}(t) & 0 \\
0 & \rho_{32}(t) & \rho_{33}(t) & 0 \\
\rho_{41}(t) & 0 & 0 & \rho_{44}(t)
\end{array}\right)
$$

where $\rho_{11}(0)=(1-a) / 3, \rho_{14}(0)=\exp (\mathrm{i} \delta) / 9, \rho_{22}(0)=\rho_{33}(0)=1 / 3, \rho_{23}(0)=\exp (i \chi) / 3$, $\rho_{32}(0)=\exp (-i \chi) / 3$, and $\rho_{44}(0)=a / 3$.

Inserting Eq.(15) and SU(4) matrix elements into Eq. (14), then using Eq. (16), the expression of the Bloch radius can be expressed as

$$
\begin{gathered}
r^{2}=\frac{2}{3}\left(\left(\rho_{23}+\rho_{32}\right)^{2}-\left(\rho_{32}-\rho_{23}\right)^{2}+\left(\rho_{22}-\rho_{33}\right)^{2}+\frac{1}{3}\left(\rho_{22}+\rho_{33}-2 \rho_{44}\right)^{2}\right. \\
\left.+\left(\rho_{41}+\rho_{14}\right)^{2}-\left(\rho_{41}-\rho_{14}\right)^{2}+\frac{1}{6}\left(1-4 \rho_{11}\right)^{2}\right)
\end{gathered}
$$

The corresponding eigenvectors $\left|\chi_{k}\right\rangle$ of $\rho_{\mathrm{X}}(t)$ can be expressed as

$$
\begin{aligned}
& \left|\chi_{1}\right\rangle=\frac{1}{N_{1}}\left(\begin{array}{llll}
0, & \rho_{22}-\rho_{33}-\rho_{x 1}, & 2 \rho_{32}, & 0
\end{array}\right)^{\mathrm{T}} \\
& \left|\chi_{2}\right\rangle=\frac{1}{N_{2}}\left(\begin{array}{llll}
0, & \rho_{22}-\rho_{33}+\rho_{x 1}, & 2 \rho_{32}, & 0
\end{array}\right)^{\mathrm{T}} \\
& \left|\chi_{3}\right\rangle=\frac{1}{N_{3}}\left(\begin{array}{llll}
\rho_{11}-\rho_{44}-\rho_{x 2}, & 0, & 0, & 2 \rho_{41}
\end{array}\right)^{\mathrm{T}} \\
& \left|\chi_{4}\right\rangle=\frac{1}{N_{4}}\left(\begin{array}{llll}
\rho_{11}-\rho_{44}+\rho_{x 2}, & 0, & 0, & 2 \rho_{41}
\end{array}\right)^{\mathrm{T}}
\end{aligned}
$$

The corresponding eigenvalues of density matrix can be written as

$$
\begin{aligned}
& \chi_{1}=\frac{1}{2}\left(\rho_{22}+\rho_{33}-\rho_{x 1}\right) \\
& \chi_{2}=\frac{1}{2}\left(\rho_{22}+\rho_{33}+\rho_{x 1}\right) \\
& \chi_{3}=\frac{1}{2}\left(\rho_{11}+\rho_{44}-\rho_{x 2}\right)
\end{aligned}
$$




$$
\chi_{4}=\frac{1}{2}\left(\rho_{11}+\rho_{44}+\rho_{x 2}\right)
$$

where

$$
\begin{aligned}
& N_{1}=\sqrt{4 \rho_{32}^{2}+\left(\rho_{22}-\rho_{33}-\rho_{x 1}\right)^{2}} \\
& N_{2}=\sqrt{4 \rho_{32}^{2}+\left(\rho_{22}-\rho_{33}+\rho_{x 1}\right)^{2}} \\
& N_{3}=\sqrt{4 \rho_{41}^{2}+\left(\rho_{11}-\rho_{44}-\rho_{x 2}\right)^{2}} \\
& N_{4}=\sqrt{4 \rho_{41}^{2}+\left(\rho_{11}-\rho_{44}+\rho_{x 2}\right)^{2}}
\end{aligned}
$$

with

$$
\begin{aligned}
& \rho_{x 1}=\sqrt{\left(\rho_{22}-\rho_{33}\right)^{2}+4 \rho_{23} \rho_{32}} \\
& \rho_{x 2}=\sqrt{\left(\rho_{11}-\rho_{44}\right)^{2}+4 \rho_{14} \rho_{41}}
\end{aligned}
$$

The time-dependent density matrix of $\mathrm{Y}$ state is written as

$$
\rho_{\mathrm{Y}}(t)=\left(\begin{array}{cccc}
\rho_{11}(t) & 0 & 0 & 0 \\
0 & \rho_{22}(t) & \rho_{23}(t) & 0 \\
0 & \rho_{32}(t) & \rho_{33}(t) & 0 \\
0 & 0 & 0 & \rho_{44}(t)
\end{array}\right)
$$

the initial parameters are same as $\mathrm{X}$ state.

The Bloch radius $r$ of Y state can be obtained by setting $\rho_{14}=\rho_{41}=0$ in Eq. (25).

The corresponding eigenvectors $\left|\chi_{k}\right\rangle$, as well as $\rho_{\mathrm{Y}}(t)$ are given by

$$
\begin{aligned}
& \left|\chi_{1}\right\rangle=\left(\begin{array}{llll}
1, & 0, & 0, & 0
\end{array}\right)^{\mathrm{T}} \\
& \left|\chi_{2}\right\rangle=\frac{1}{N_{1}}\left(\begin{array}{lll}
0, & \rho_{22}-\rho_{33}-\rho_{x 1}, \quad 2 \rho_{32}, \quad 0
\end{array}\right)^{\mathrm{T}} \\
& \left|\chi_{3}\right\rangle=\frac{1}{N_{2}}\left(\begin{array}{lll}
0, & \rho_{22}-\rho_{33}+\rho_{x 1}, \quad 2 \rho_{32}, \quad 0
\end{array}\right)^{\mathrm{T}} \\
& \left|\chi_{4}\right\rangle=\left(\begin{array}{llll}
0, & 0, & 0, & 1
\end{array}\right)^{\mathrm{T}}
\end{aligned}
$$


The eigenvalues of density matrix can be written as

$$
\begin{aligned}
& \chi_{1}=\rho_{11} \\
& \chi_{2}=\frac{1}{2}\left(\rho_{22}+\rho_{33}-\rho_{x 1}\right) \\
& \chi_{3}=\frac{1}{2}\left(\rho_{22}+\rho_{33}+\rho_{x 1}\right) \\
& \chi_{4}=\rho_{44}
\end{aligned}
$$

where $\rho_{x 1}, N_{1}$ and $N_{2}$ are the same as $\mathrm{X}$ state.

\section{Density matrix of mixed states}

In the situations of $\omega_{\mathrm{A}}=\omega_{\mathrm{B}}=\omega$ and $\chi_{\mathrm{r}, \mathrm{A}}=\chi_{\mathrm{r}, \mathrm{B}}=\chi_{\mathrm{r}}$ as well as $\Gamma_{\mathrm{A}}=\Gamma_{\mathrm{B}}=\Gamma$ and $\gamma_{\mathrm{A}}=$ $=\gamma_{\mathrm{B}}=\gamma$, the master Eq. (5) with Eq. (6) of density matrix may expressed as component forms, i.e.,

$$
\begin{aligned}
\dot{\rho}_{11}(t)= & -2 n \gamma \rho_{11}(t) \\
\dot{\rho}_{14}(t)= & -n \gamma \rho_{14}(t)-2 i \omega \rho_{14}(t)-2\left(\Gamma+\Gamma_{0}\right) \rho_{14}(t) \\
\dot{\rho}_{21}(t)= & -\frac{1}{2} n\left(3 \gamma \rho_{21}(t)+\Gamma \rho_{31}(t)\right) \\
& -i\left(J \rho_{31}(t)-\rho_{21}(t)\left(\omega-2 n \chi_{\mathrm{r}}\right)\right)-\Gamma \rho_{21}(t) \\
\dot{\rho}_{22}(t)= & i J\left(\rho_{23}(t)-\rho_{32}(t)\right) \\
& +\frac{1}{2} n\left(2 \gamma \rho_{11}(t)-2 \gamma \rho_{22}(t)-\Gamma\left(\rho_{23}(t)+\rho_{32}(t)\right)\right) \\
\dot{\rho}_{23}(t)= & -n \gamma \rho_{23}(t)-\frac{1}{2} n \Gamma\left(-2 \rho_{11}(t)+\rho_{22}(t)+\rho_{33}(t)\right) \\
& +i\left(J \rho_{22}(t)-J \rho_{33}(t)-4 n \rho_{23}(t) \chi_{\mathrm{r}}\right)+2\left(\Gamma_{0}-\Gamma\right) \rho_{23}(t) \\
\dot{\rho}_{32}(t)= & -n \gamma \rho_{32}(t)-\frac{1}{2} n \Gamma\left(-2 \rho_{11}(t)+\rho_{22}(t)+\rho_{33}(t)\right) \\
& -i\left(J \rho_{22}(t)-J \rho_{33}(t)-4 n \rho_{32}(t) \chi_{\mathrm{r}}\right)+2\left(\Gamma_{0}-\Gamma\right) \rho_{32}(t)
\end{aligned}
$$




$$
\begin{aligned}
\dot{\rho}_{33}(t)= & -i J\left(\rho_{23}(t)-\rho_{32}(t)\right) \\
& +\frac{1}{2} n\left(2 \gamma \rho_{11}(t)-2 \gamma \rho_{33}(t)-\Gamma\left(\rho_{23}(t)+\rho_{32}(t)\right)\right) \\
\dot{\rho}_{41}(t)= & -n \gamma \rho_{41}(t)+2 i \omega \rho_{41}(t)-2\left(\Gamma+\Gamma_{0}\right) \rho_{41}(t) \\
\dot{\rho}_{44}(t)= & n\left(\gamma \rho_{22}(t)+\Gamma \rho_{23}(t)+\Gamma \rho_{32}(t)+\gamma \rho_{33}(t)\right)
\end{aligned}
$$

In order to get an analytic solution of Eqs. (49)-(57), we assume that

$$
\begin{aligned}
& X=\left(\rho_{23}(t)+\rho_{32}(t)\right) \exp (2 n \gamma t) \\
& Y=\left(\rho_{23}(t)-\rho_{32}(t)\right) \exp (2 n \gamma t) \\
& Z=\left(\rho_{22}(t)+\rho_{33}(t)\right) \exp (2 n \gamma t) \\
& W=\left(\rho_{22}(t)-\rho_{33}(t)\right) \exp (2 n \gamma t)
\end{aligned}
$$

and the matrix form is written as

$$
\frac{\mathrm{d}}{\mathrm{d} t}\left(\begin{array}{c}
X \\
Y \\
Z \\
W
\end{array}\right)=\left(\begin{array}{cccc}
2\left(\Gamma_{0}-\Gamma\right)+n \gamma & -4 i n \chi_{\mathrm{r}} & -n \Gamma & 0 \\
-4 i n \chi_{\mathrm{r}} & 2\left(\Gamma_{0}-\Gamma\right)+n \gamma & 0 & 2 i J \\
-n \Gamma & 0 & n \gamma & 0 \\
0 & 2 i J & 0 & n \gamma
\end{array}\right)\left(\begin{array}{l}
X \\
Y \\
Z \\
W
\end{array}\right)+\left(\begin{array}{c}
\frac{2 n \Gamma(1-a)}{3} \\
0 \\
0 \\
\frac{2 n \gamma(1-a)}{3}
\end{array}\right)
$$

In order to cancel the constant term, we set

$$
\begin{aligned}
& X=X^{\prime}+X^{\prime \prime} \\
& Y=Y^{\prime}+Y^{\prime \prime} \\
& Z=Z^{\prime}+Z^{\prime \prime} \\
& W=W^{\prime}+W^{\prime \prime}
\end{aligned}
$$

which satisfy 


$$
\left(\begin{array}{cccc}
2\left(\Gamma_{0}-\Gamma\right)+n \gamma & -4 i n \chi_{\mathrm{r}} & -n \Gamma & 0 \\
-4 i n \chi_{\mathrm{r}} & 2\left(\Gamma_{0}-\Gamma\right)+n \gamma & 0 & 2 i J \\
-n \Gamma & 0 & n \gamma & 0 \\
0 & 2 i J & 0 & n \gamma
\end{array}\right)\left(\begin{array}{l}
X^{\prime \prime} \\
Y^{\prime \prime} \\
Z^{\prime \prime} \\
W^{\prime \prime}
\end{array}\right)+\left(\begin{array}{c}
\frac{2 n \Gamma(1-a)}{3} \\
0 \\
0 \\
\frac{2 n \gamma(1-a)}{3}
\end{array}\right)=0
$$

The parameters of $X^{\prime \prime}, Y^{\prime \prime}, Z^{\prime \prime}, W^{\prime \prime}$ can be obtained by calculating the matrix equation above, and the specific results are in the Appendix II.

Thus Eq. (62) can be rewritten as

$$
\frac{\mathrm{d}}{\mathrm{d} t}\left(\begin{array}{c}
X \\
Y \\
Z \\
W
\end{array}\right)=\frac{\mathrm{d}}{\mathrm{d} t}\left(\begin{array}{l}
X^{\prime} \\
Y^{\prime} \\
Z^{\prime} \\
W^{\prime}
\end{array}\right)=\left(\begin{array}{cccc}
2\left(\Gamma_{0}-\Gamma\right)+n \gamma & -4 i n \chi_{\mathrm{r}} & -n \Gamma & 0 \\
-4 i n \chi_{\mathrm{r}} & 2\left(\Gamma_{0}-\Gamma\right)+n \gamma & 0 & 2 i J \\
-n \Gamma & 0 & n \gamma & 0 \\
0 & 2 i J & 0 & n \gamma
\end{array}\right)\left(\begin{array}{l}
X^{\prime} \\
Y^{\prime} \\
Z^{\prime} \\
W^{\prime}
\end{array}\right)
$$

The solution to Eq. (68) may be constructed by

$$
\begin{array}{r}
\left(\begin{array}{c}
X^{\prime} \\
Y^{\prime} \\
Z^{\prime} \\
W^{\prime}
\end{array}\right)=c_{1} \exp \left(x_{1} t\right)\left(\begin{array}{c}
h_{1} \\
i \frac{n \gamma-x_{1}}{2 J} \\
h_{2} \\
1
\end{array}\right)+c_{2} \exp \left(x_{2} t\right)\left(\begin{array}{c}
h_{3} \\
i \frac{n \gamma-x_{2}}{2 J} \\
h_{4} \\
1
\end{array}\right) \\
+\left(c_{\mathrm{r}}+i c_{\mathrm{i}}\right) \exp \left(\left(x_{\mathrm{r}}+i x_{\mathrm{i}}\right) t\right)\left(\begin{array}{c}
h_{\mathrm{r}}+i h_{\mathrm{i}} \\
i \frac{n \gamma-x_{\mathrm{r}}-i x_{\mathrm{i}}}{2 J} \\
\beta_{\mathrm{r}}+i \beta_{\mathrm{i}} \\
1
\end{array}\right) \\
+\left(c_{\mathrm{r}}-i c_{\mathrm{i}}\right) \exp \left(\left(x_{\mathrm{r}}-i x_{\mathrm{i}}\right) t\right)\left(\begin{array}{c}
h_{\mathrm{r}}-i h_{\mathrm{i}} \\
i \frac{n \gamma-x_{\mathrm{r}}+i x_{\mathrm{i}}}{2 J} \\
\beta_{\mathrm{r}}-i \beta_{\mathrm{i}} \\
1
\end{array}\right)
\end{array}
$$


or it can be written as a system of equations

$$
\begin{aligned}
X^{\prime}= & c_{1} \exp \left(x_{1} t\right) h_{1}+c_{2} \exp \left(x_{2} t\right) h_{3}+2 c_{\mathrm{r}} h_{\mathrm{r}} \cos \left(x_{\mathrm{i}} t\right) \exp \left(x_{\mathrm{r}} t\right) \\
& -2 c_{\mathrm{i}} h_{\mathrm{i}} \cos \left(x_{\mathrm{i}} t\right) \exp \left(x_{\mathrm{r}} t\right)-2 c_{\mathrm{r}} h_{\mathrm{i}} \sin \left(x_{\mathrm{i}} t\right) \exp \left(x_{\mathrm{r}} t\right) \\
& -2 c_{\mathrm{i}} h_{\mathrm{r}} \sin \left(x_{\mathrm{i}} t\right) \exp \left(x_{\mathrm{r}} t\right) \\
Y^{\prime}= & \frac{i}{2 J}\left(c_{1} \exp \left(x_{1} t\right)\left(n \gamma-x_{1}\right)+c_{2} \exp \left(x_{2} t\right)\left(n \gamma-x_{2}\right)\right. \\
& +2 c_{\mathrm{r}} \cos \left(x_{\mathrm{i}} t\right) \exp \left(x_{\mathrm{r}} t\right)\left(n \gamma-x_{\mathrm{r}}\right)-2 c_{\mathrm{i}} x_{\mathrm{i}} \cos \left(x_{\mathrm{i}} t\right) \exp \left(x_{\mathrm{r}} t\right) \\
& \left.+2 c_{\mathrm{r}} x_{\mathrm{i}} \sin \left(x_{\mathrm{i}} t\right) \exp \left(x_{\mathrm{r}} t\right)-2 c_{\mathrm{i}} \sin \left(x_{\mathrm{i}} t\right) \exp \left(x_{\mathrm{r}} t\right)\left(n \gamma-x_{\mathrm{r}}\right)\right) \\
Z^{\prime}= & c_{1} \exp \left(x_{1} t\right) h_{2}+c_{2} \exp \left(x_{2} t\right) h_{4}+2 c_{\mathrm{r}} \cos \left(x_{\mathrm{i}} t\right) \exp \left(x_{\mathrm{r}} t\right) \beta_{\mathrm{r}} \\
& -2 c_{\mathrm{i}} \cos \left(x_{\mathrm{i}} t\right) \exp \left(x_{\mathrm{r}} t\right) \beta_{\mathrm{i}}-2 c_{\mathrm{r}} \sin \left(x_{\mathrm{i}} t\right) \exp \left(x_{\mathrm{r}} t\right) \beta_{\mathrm{i}} \\
& -2 c_{\mathrm{i}} \sin \left(x_{\mathrm{i}} t\right) \exp \left(x_{\mathrm{r}} t\right) \beta_{\mathrm{r}} \\
W^{\prime}= & c_{1} \exp \left(x_{1} t\right)+c_{2} \exp \left(x_{2} t\right)+2 c_{\mathrm{r}} \cos \left(x_{\mathrm{i}} t\right) \exp \left(x_{\mathrm{r}} t\right) \\
& -2 c_{\mathrm{i}} \sin \left(x_{\mathrm{i}} t\right) \exp \left(x_{\mathrm{r}} t\right)
\end{aligned}
$$

The parameters of $c_{1}, c_{2}, c_{\mathrm{r}}, c_{\mathrm{i}}, x_{1}, x_{2}, x_{\mathrm{r}}, x_{\mathrm{i}}, h_{1}, h_{2}, h_{3}, h_{4}, h_{\mathrm{r}}, h_{\mathrm{i}}, \beta_{\mathrm{r}}, \beta_{\mathrm{i}}$ can be obtained by plugging in the initial values which are shown in Appendix II.

Thus the analytical solutions of the master Eq. (5) can be expressed as

$$
\begin{aligned}
\rho_{11}(t)= & \frac{1-a}{3} \exp (-2 n \gamma t) \\
\rho_{14}(t)= & \frac{1}{9} \exp (i \delta) \exp \left(-\left(n \gamma+2 \Gamma+2 \Gamma_{0}+2 i \omega\right) t\right) \\
\rho_{22}(t)= & \frac{1}{2}\left(c_{1} \exp \left(x_{1} t\right)\left(h_{2}+1\right)+c_{2} \exp \left(x_{2} t\right)\left(h_{4}+1\right)\right. \\
& +2 c_{\mathrm{r}} \cos \left(x_{\mathrm{i}} t\right) \exp \left(x_{\mathrm{r}} t\right)\left(\beta_{\mathrm{r}}+1\right)-2 c_{\mathrm{i}} \cos \left(x_{\mathrm{i}} t\right) \exp \left(x_{\mathrm{r}} t\right) \beta_{\mathrm{i}} \\
& \left.-2 c_{\mathrm{r}} \sin \left(x_{\mathrm{i}} t\right) \exp \left(x_{\mathrm{r}} t\right) \beta_{\mathrm{i}}-2 c_{\mathrm{i}} \sin \left(x_{\mathrm{i}} t\right) \exp \left(x_{\mathrm{r}} t\right)\left(\beta_{\mathrm{i}}+1\right)+Z^{\prime \prime}+W^{\prime \prime}\right) \\
& \times \exp (-2 n \gamma t) \\
\rho_{23}(t)= & \frac{1}{2}\left(\left(c_{1} \exp \left(x_{1} t\right) h_{1}+c_{2} \exp \left(x_{2} t\right) h_{3}+2 c_{\mathrm{r}} h_{\mathrm{r}} \cos \left(x_{\mathrm{i}} t\right) \exp \left(x_{\mathrm{r}} t\right)\right.\right. \\
& -2 c_{\mathrm{i}} h_{\mathrm{i}} \cos \left(x_{\mathrm{i}} t\right) \exp \left(x_{\mathrm{r}} t\right)-2 c_{\mathrm{r}} h_{\mathrm{i}} \sin \left(x_{\mathrm{i}} t\right) \exp \left(x_{\mathrm{r}} t\right)
\end{aligned}
$$




$$
\begin{aligned}
& \left.-2 c_{\mathrm{i}} h_{\mathrm{r}} \sin \left(x_{\mathrm{i}} t\right) \exp \left(x_{\mathrm{r}} t\right)\right)+\frac{i}{2 J}\left(c_{1} \exp \left(x_{1} t\right)\left(n \gamma-x_{1}\right)\right. \\
& +c_{2} \exp \left(x_{2} t\right)\left(n \gamma-x_{2}\right)+2 c_{\mathrm{r}} \cos \left(x_{\mathrm{i}} t\right) \exp \left(x_{\mathrm{r}} t\right)\left(n \gamma-x_{\mathrm{r}}\right) \\
& +2 c_{\mathrm{i}} x_{\mathrm{i}} \cos \left(x_{\mathrm{i}} t\right) \exp \left(x_{\mathrm{r}} t\right)+2 c_{\mathrm{r}} x_{\mathrm{i}} \sin \left(x_{\mathrm{i}} t\right) \exp \left(x_{\mathrm{r}} t\right) \\
& \left.\left.-2 c_{\mathrm{i}} \sin \left(x_{\mathrm{i}} t\right) \exp \left(x_{\mathrm{r}} t\right)\left(n \gamma-x_{\mathrm{r}}\right)\right)+X^{\prime \prime}+Y^{\prime \prime}\right) \exp (-n \gamma t) \\
& \rho_{32}(t)=\frac{1}{2}\left(\left(c_{1} \exp \left(x_{1} t\right) h_{1}+c_{2} \exp \left(x_{2} t\right) h_{3}+2 c_{\mathrm{r}} h_{\mathrm{r}} \cos \left(x_{\mathrm{i}} t\right) \exp \left(x_{\mathrm{r}} t\right)\right.\right. \\
& -2 c_{\mathrm{i}} h_{\mathrm{i}} \cos \left(x_{\mathrm{i}} t\right) \exp \left(x_{\mathrm{r}} t\right)-2 c_{\mathrm{r}} h_{\mathrm{i}} \sin \left(x_{\mathrm{i}} t\right) \exp \left(x_{\mathrm{r}} t\right) \\
& \left.-2 c_{\mathrm{i}} h_{\mathrm{r}} \sin \left(x_{\mathrm{i}} t\right) \exp \left(x_{\mathrm{r}} t\right)\right)-\frac{i}{2 J}\left(c_{1} \exp \left(x_{1} t\right)\left(n \gamma-x_{1}\right)\right. \\
& +c_{2} \exp \left(x_{2} t\right)\left(n \gamma-x_{2}\right)+2 c_{\mathrm{r}} \cos \left(x_{\mathrm{i}} t\right) \exp \left(x_{\mathrm{r}} t\right)\left(n \gamma-x_{\mathrm{r}}\right) \\
& +2 c_{\mathrm{i}} x_{\mathrm{i}} \cos \left(x_{\mathrm{i}} t\right) \exp \left(x_{\mathrm{r}} t\right)+2 c_{\mathrm{r}} x_{\mathrm{i}} \sin \left(x_{\mathrm{i}} t\right) \exp \left(x_{\mathrm{r}} t\right) \\
& \left.\left.-2 c_{\mathrm{i}} \sin \left(x_{\mathrm{i}} t\right) \exp \left(x_{\mathrm{r}} t\right)\left(n \gamma-x_{\mathrm{r}}\right)\right)+X^{\prime \prime}+Y^{\prime \prime}\right) \exp (-n \gamma t) \\
& \rho_{33}(t)=\frac{1}{2}\left(c_{1} \exp \left(x_{1} t\right)\left(h_{2}-1\right)+c_{2} \exp \left(x_{2} t\right)\left(h_{4}-1\right)\right. \\
& +2 c_{\mathrm{r}} \cos \left(x_{\mathrm{i}} t\right) \exp \left(x_{\mathrm{r}} t\right)\left(\beta_{\mathrm{r}}-1\right)-2 c_{\mathrm{i}} \cos \left(x_{\mathrm{i}} t\right) \exp \left(x_{\mathrm{r}} t\right) \beta_{\mathrm{i}} \\
& \left.-2 c_{\mathrm{r}} \sin \left(x_{\mathrm{i}} t\right) \exp \left(x_{\mathrm{r}} t\right) \beta_{\mathrm{i}}-2 c_{\mathrm{i}} \sin \left(x_{\mathrm{i}} t\right) \exp \left(x_{\mathrm{r}} t\right)\left(\beta_{\mathrm{r}}-1\right)+Z^{\prime \prime}-W^{\prime \prime}\right) \\
& \times \exp (-2 n \gamma t) \\
& \rho_{41}(t)=\frac{1}{9} \exp (-i \delta) \exp (-(n \gamma+2 \Gamma-2 i \omega) t) \\
& \rho_{44}(t)=1-\rho_{11}(t)-\rho_{22}(t)-\rho_{33}(t)
\end{aligned}
$$

\section{Nonlocal quantum correlations of two-qubit system}

Besides the quantum geometric phase, another fascinating phenomenon is nonlocal quantum correlation in nature. The idea of quantum nonlocality among remote particles was originally exploited by EINSTEIN et al. [41], further conceptualized in a seminal paper by SCHRÖDINGER [42], and a subsequent work by BELL [43].

At present, there exist two kinds of nonlocal quantum correlations, i.e., quantum entanglement and quantum discord. An entangled state is a state of a composite system that cannot be separated into product states in terms of the subsystems. But the quantum 
entanglement is not a unique kind of useful correlation emerging in quantum many-particle system. Even though the entanglement is subtracted from total quantum correlations, the residual correlations are not of an entirely classical origin. Such a correlation, called as quantum discord, is useful to measure the nonclassical correlations that cannot be captured by entanglement in the quantum many-particle system.

Quantum entanglement can be quantified by the concurrence $[\underline{2}, \underline{13}]$, i.e.,

$$
C(t)=\max \{0, \tilde{C}(t)\}
$$

where $\tilde{C}(t)=\sqrt{\lambda_{1}}-\sqrt{\lambda_{2}}-\sqrt{\lambda_{3}}-\sqrt{\lambda_{4}}$ and the quantities $\lambda_{j}(j=1,2,3,4)$ are the eigenvalues arranged in a decreasing order of the following matrix,

$$
\rho^{\prime}(t)=\rho(t)\left(\sigma_{1}^{y} \otimes \sigma_{2}^{y}\right) \rho^{*}(t)\left(\sigma_{1}^{y} \otimes \sigma_{2}^{y}\right)
$$

where $\rho^{*}(t)$ is a complex conjugation of $\rho(t)$ and $\sigma^{y}$ is a Pauli matrix of $y$-direction. It is noted that the concurrence varies from $C=0$ for a completely unentangled state to $C=1$ for a maximally entangled state.

Inserting Eq. (24) into Eq. (83), we can obtain the eigenvalues of $\rho^{\prime}(t)$. For the $\mathrm{X}$-state, thus, one has

$$
\tilde{C}(t)=2 \max \left(0,\left|\rho_{23}\right|-\sqrt{\rho_{11} \rho_{44}},\left|\rho_{14}\right|-\sqrt{\rho_{22} \rho_{33}}\right)
$$

with the conditions $\rho_{22} \rho_{33} \geq\left|\rho_{23}\right|^{2}, \rho_{11} \rho_{44} \geq\left|\rho_{14}\right|^{2}$ and $\Sigma_{i} \rho_{i i}=1$.

For the Y-state, similarly, we find

$$
\tilde{C}(t)=2 \max \left(0,\left|\rho_{23}\right|-\sqrt{\rho_{11} \rho_{44}}\right)
$$

in terms of Eqs. (40), (82) and (83).

In order to describe the dynamic evolution of quantum correlations, we first consider mutual information. The mutual information is an approach to measure total quantum and classical correlations [ $[\underline{4}, \underline{45}]$,

$$
I\left(\rho_{\mathrm{AB}}\right)=S\left(\rho_{\mathrm{A}}\right)+S\left(\rho_{\mathrm{B}}\right)-S\left(\rho_{\mathrm{AB}}\right)
$$

where $S\left(\rho_{\mathrm{A}}\right)=H\left(\rho_{11}+\rho_{22}\right), S\left(\rho_{\mathrm{B}}\right)=H\left(\rho_{11}+\rho_{33}\right)$, and

$$
S\left(\rho_{\mathrm{AB}}\right)=-\sum_{i=0}^{4} \chi_{i} \log _{2} \chi_{i}
$$

are von Neumann entropies with the relation $H(x)=-x \log _{2} x-(1-x) \log _{2}(1-x)$.

Next, a set of complete basis vectors, $\left\{B_{k}, k=1,2\right\}$, is introduced to measure the subsystem $B$, and the post-measured state can be written as

$$
\rho_{k}=\frac{1}{p_{k}}\left(I_{\mathrm{A}} \otimes B_{k}\right) \rho_{\mathrm{AB}}\left(I_{\mathrm{A}} \otimes B_{k}\right)
$$


where $p_{k}=\operatorname{Tr}\left(\left(I_{\mathrm{A}} \otimes B_{k}\right) \rho_{\mathrm{AB}}\left(I_{\mathrm{A}} \otimes B_{k}\right)\right)$, and $I_{\mathrm{A}}$ is an identity operator on the subsystem $A$, then any von Neumann measurement for the subsystem $B$ can be written as

$$
B_{k}=V \Pi_{k} V^{\dagger}: k=0,1
$$

with the unitary matrix $V=t+i \mathbf{y} \cdot \boldsymbol{\sigma} \in U(2)$ for $t \in \mathbb{R}$ and $\mathbf{y}=\left(y_{1}, y_{2}, y_{3}\right) \in \mathbb{R}^{3}$ [19] , and $\Pi_{k}=|k\rangle\langle k|(k=0,1)$.

The classical correlation can be expressed as

$$
\begin{aligned}
\Lambda\left(\rho_{\mathrm{AB}}\right) & =\max _{\left\{B_{k}\right\}} I\left(\rho_{\mathrm{AB}} \mid\left\{B_{k}\right\}\right)=\max _{\left\{B_{k}\right\}}\left(S\left(\rho_{\mathrm{A}}\right)-\sum_{k} p_{k} S\left(\rho_{k}\right)\right) \\
& =S\left(\rho_{\mathrm{A}}\right)-\min _{\left\{B_{k}\right\}}\left(\sum_{k} p_{k} S\left(\rho_{k}\right)\right)
\end{aligned}
$$

where $I\left(\rho_{\mathrm{AB}} \mid\left\{B_{k}\right\}\right)=S\left(\rho_{\mathrm{A}}\right)-\Sigma_{k} p_{k} S\left(\rho_{k}\right)$ are used [ $\underline{46}, \underline{47]}$.

The quantum discord $[\underline{3}, \underline{4}, \underline{46}, \underline{47}]$ is defined by

$$
D\left(\rho_{\mathrm{AB}}\right)=I\left(\rho_{\mathrm{AB}}\right)-\Lambda\left(\rho_{\mathrm{AB}}\right)
$$

Inserting Eqs. (86), (90) into Eq. (91), the $D\left(\rho_{\mathrm{AB}}\right)$ can be expressed as

$$
D\left(\rho_{\mathrm{AB}}\right)=\min _{\left\{B_{k}\right\}}\left(S\left(\rho_{\mathrm{B}}\right)-S\left(\rho_{\mathrm{AB}}\right)-\sum_{k} p_{k} S\left(\rho_{k}\right)\right)
$$

which can further be expressed as

$$
D\left(\rho_{\mathrm{AB}}\right)=\min \left\{D_{1}\left(\rho_{\mathrm{AB}}\right), D_{2}\left(\rho_{\mathrm{AB}}\right)\right\}
$$

where

$$
\begin{aligned}
& D_{1}\left(\rho_{\mathrm{AB}}\right)=S\left(\rho_{\mathrm{B}}\right)+S\left(\rho_{\mathrm{AB}}\right)-M \\
& D_{2}\left(\rho_{\mathrm{AB}}\right)=S\left(\rho_{\mathrm{B}}\right)+S\left(\rho_{\mathrm{AB}}\right)-N
\end{aligned}
$$

with $M=\Sigma_{i} \rho_{i i} \log _{2} \rho_{i i}+H\left(\rho_{11}+\rho_{33}\right)$ and $N=-H(\tau)$.

For the $\mathrm{X}$-state, we find

$$
\tau=\frac{1}{2}\left(1+\sqrt{\left(1-2\left(\rho_{33}+\rho_{44}\right)\right)^{2}+4\left(\left|\rho_{14}\right|+\left|\rho_{23}\right|\right)^{2}}\right)
$$

and for the Y-state, we have

$$
\tau=\frac{1}{2}\left(1+\sqrt{\left(1-2\left(\rho_{33}+\rho_{44}\right)\right)^{2}+4\left|\rho_{23}\right|^{2}}\right)
$$




\section{Discussions}

It is known that the quantum nonlocality can be described by the concurrence, the quantum discord and the geometric phase, respectively. On the other hand, the Bloch radius describes a mixture degree of the single-particle physical system. Therefore, it is interesting to distinguish and compare them in all cases as shown in Figs. 2-8.

In Fig. 2 for the X state, we find that with changing the phonon numbers the $n$-th concurrence and quantum discord show an initial dip followed by enhancement of the correlations and then the decays at a long time. The larger the phonon number is, the greater the effects of enhancement for the correlations are at the beginning, but the shorter the decay time is. It implies that, when the phonon number increases, there will be an increasing coherence, but evolving with the time, it will easily go to zero. In other words, the ability to retain the retreat is weakened. However, the Pancharatnam phase would decrease, and the larger $n$ is, the greater the rate of the decrease is.

It is interesting to observe the Bloch radius for the two-particle system, where the Bloch radius first decreases and then increases, and finally approaches a value less than one as opposed to a single-particle system. From the derived Bloch radius Eq. (25), we can see that the last term is $\left(1-\rho_{11}\right)^{2} / 6$, and the matrix element $\rho_{11}$ is a decreasing function of the time and finally approaches to 0 . Therefore, the increase at the beginning is because the decay items of the previous items are strong, and then the increase is the last one. The situation is different from the single-particle system.
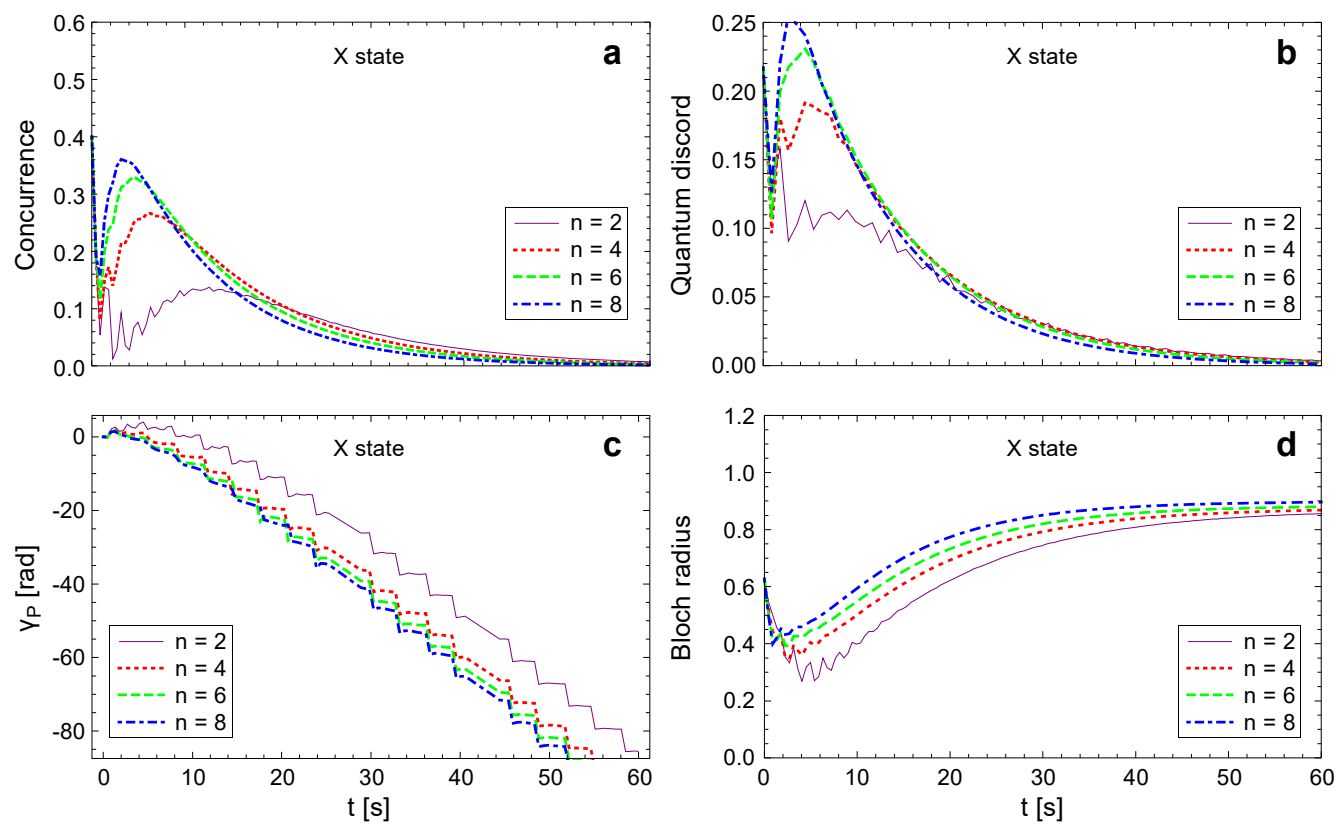

Fig. 2. Different phonon numbers $n$ for concurrence (a), quantum discord (b), Pancharatnam phase (c), and Bloch radius (d) as a function of evolving time in the $\mathrm{X}$ state with the same initial conditions $J=2$, $\Gamma=0.1 \mathrm{~s}^{-1}, \gamma=0.05 \mathrm{~s}^{-1}, \Gamma_{0}=0.01 \mathrm{~s}^{-1}, a=0.2, \delta=\pi / 3, \omega=1 \mathrm{~s}^{-1}, \chi_{\mathrm{r}}=0.025$ and initial phase $\chi=3 \pi / 2$. 

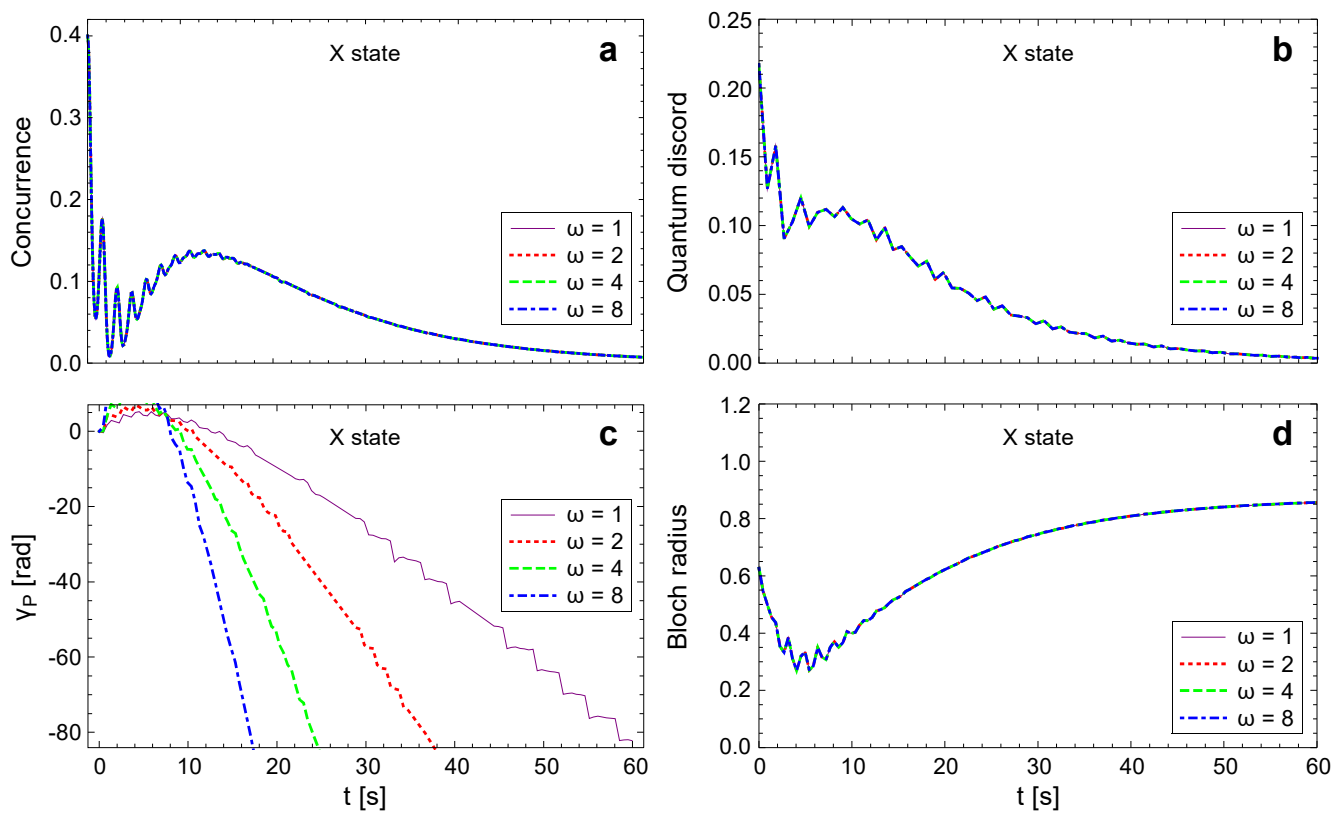

Fig. 3. Different frequencies $\omega$ for concurrence (a), quantum discord (b), Pancharatnam phase (c), and Bloch radius (d) as a function of evolving time in the $\mathrm{X}$ state with the same initial conditions $J=2$, $\Gamma=0.1 \mathrm{~s}^{-1}, \gamma=0.05 \mathrm{~s}^{-1}, \Gamma_{0}=0.01 \mathrm{~s}^{-1}, n=2, a=0.2, \delta=\pi / 3, \chi_{\mathrm{r}}=0.025$ and initial phase $\chi=3 \pi / 2$.
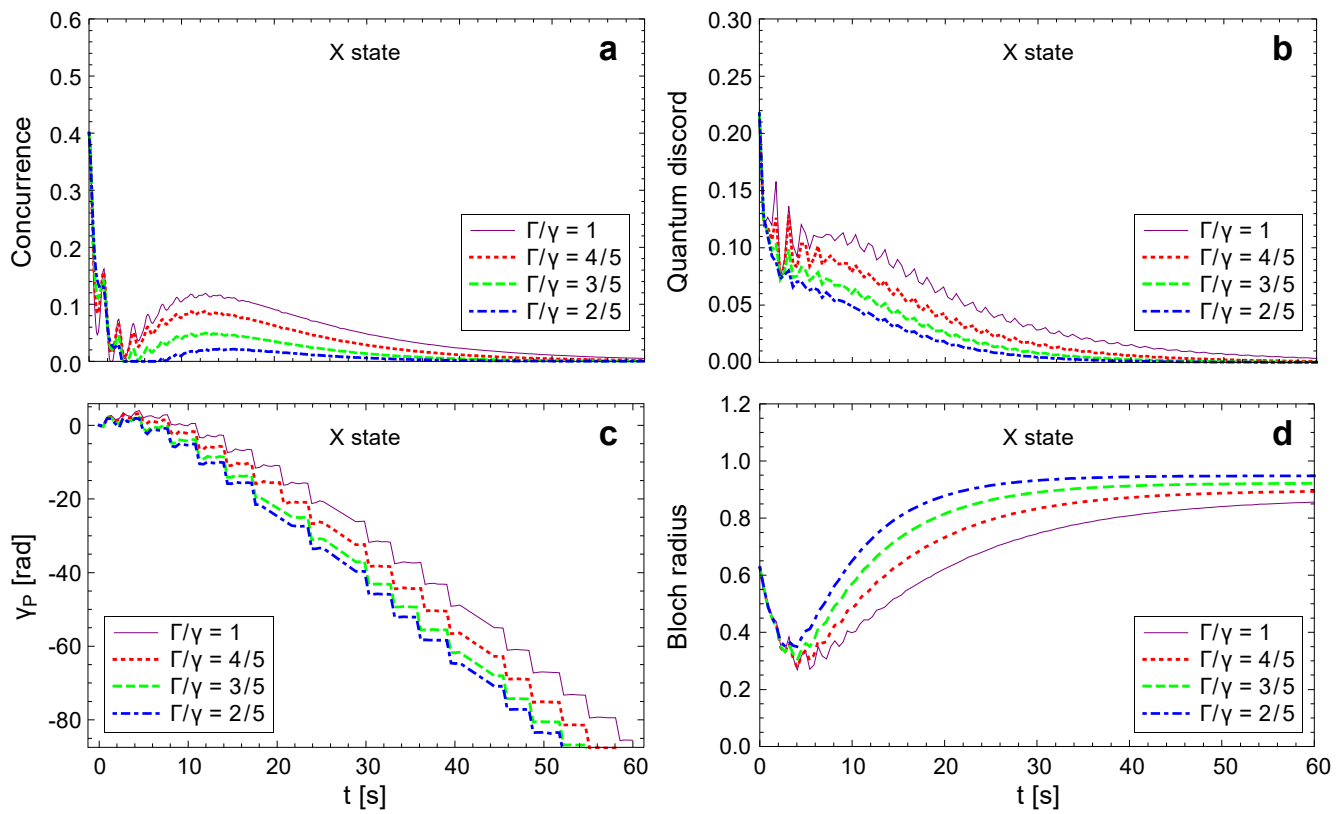

Fig. 4. Different ratios $\Gamma / \gamma$ of environments for concurrence (a), quantum discord (b), Pancharatnam phase (c), and Bloch radius $(\mathbf{d})$ as a function of evolving time in the $\mathrm{X}$ state with the same initial conditions $J=2, \Gamma_{0}=0.01 \mathrm{~s}^{-1}, \gamma=0.05 \mathrm{~s}^{-1}, n=2, a=0.2, \delta=\pi / 3, \omega=1 \mathrm{~s}^{-1}, \chi_{\mathrm{r}}=0.025$, and initial phase $\chi=3 \pi / 2$. 
For different $\omega$ shown in Fig. 3, we find that beside the oscillations, the quantum concurrence and discord as well as the Bloch radius are independent of the frequency $\omega$. But the Pancharatnam phase decreases with the increase in the frequency. In other words, the geometric phase can be used to observe such a change in the frequency.

In order to investigate into the effects of environment correlation to the quantum nonlocality, we plot the evolving curves of the concurrence, the quantum discord and the geometric phase for different ratios of the instantaneous decay $\Gamma$ to the background $\gamma$ in Fig. 4 and for the different ratios of the dephasing decay $\Gamma_{0}$ to the background $\gamma$ in Fig. 5. We find that, when the ratio of the instantaneous decay $\Gamma$ to the background $\gamma$ changes, the concurrence will still decrease at the finial time, increase and decrease at last, but the quantum discord will directly decrease with the decrease in the ratio, and keeps all the times. When the ratio is reduced, there will emerge the death and recovery phenomena of the concurrence, and the ability to retain the decoherence is getting weaker.

For the ratio of the dephasing decay $\Gamma_{0}$ to the background $\gamma$, we found that when the ratio is of a sufficient value, i.e., $1 / 2$, it is different from the small ratio values. The involving curves for all physical quantities are close to each other as shown in Eq. (6), but when the ratio is $1 / 2$, the concurrence and quantum discord increase to a larger value and take longer to go to 0 , which means that the larger the ratio is, the better the coherence is. Especially, the geometric phase has the same behavior.
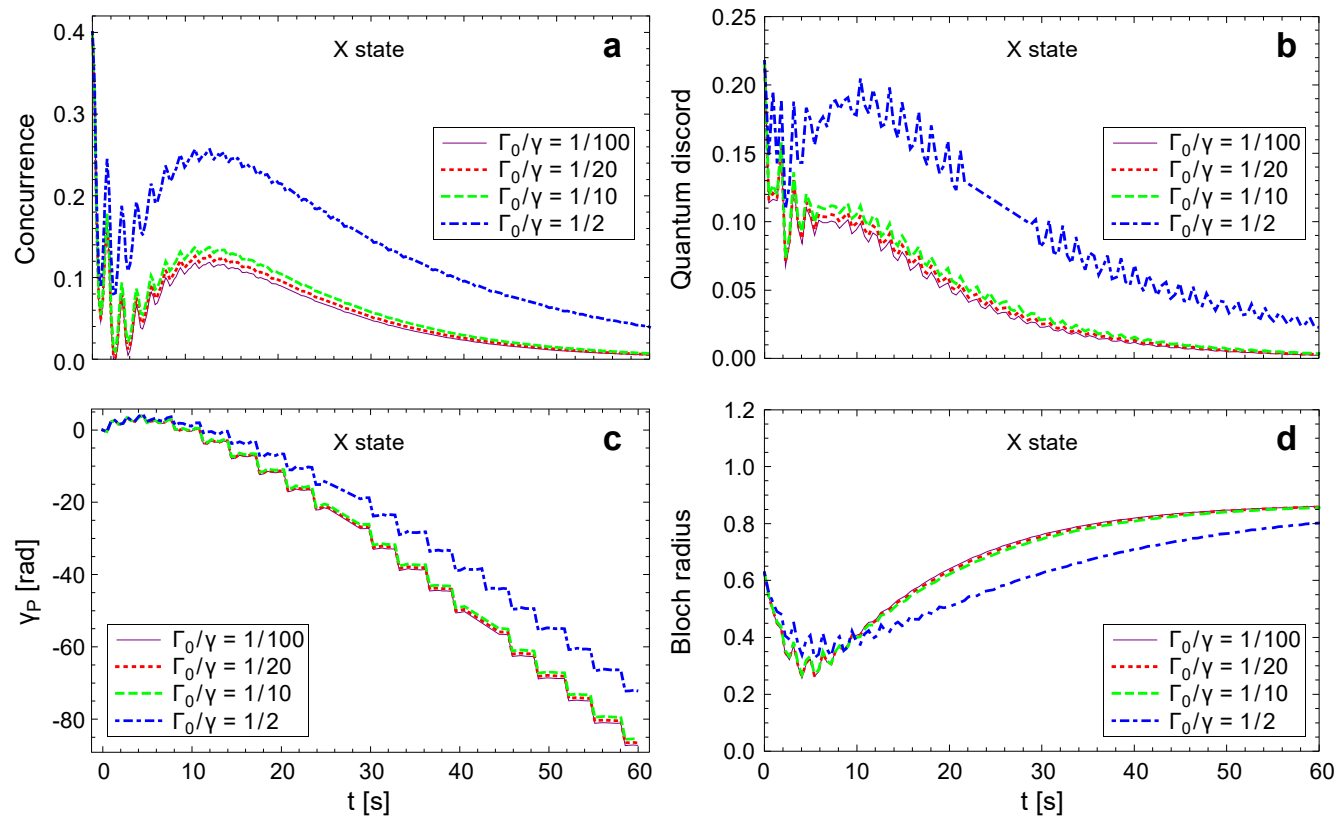

Fig. 5. Different ratios $\Gamma_{0} / \gamma$ of environments for concurrence (a), quantum discord (b), Pancharatnam phase (c), and Bloch radius (d) as a function of evolving time in the $\mathrm{X}$ state with the same initial conditions $J=2, \Gamma=0.1 \mathrm{~s}^{-1}, \gamma=0.05 \mathrm{~s}^{-1}, n=2, a=0.2, \delta=\pi / 3, \omega=1 \mathrm{~s}^{-1}, \chi_{\mathrm{r}}=0.025$, and initial phase $\chi=3 \pi / 2$. 

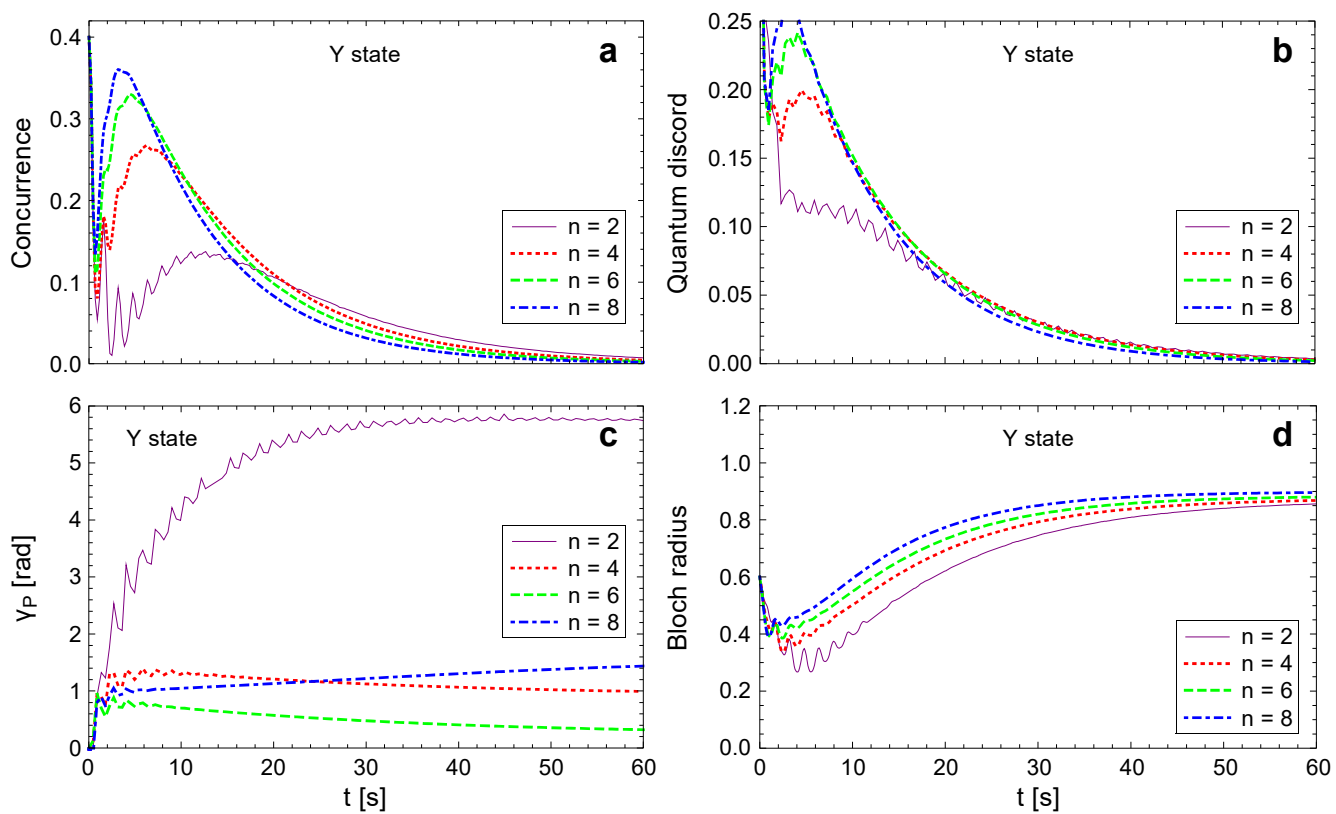

Fig. 6. Different phonon numbers $n$ for concurrence (a), quantum discord (b), Pancharatnam phase (c), and Bloch radius (d) as a function of evolving time in the $\mathrm{Y}$ state with the same initial conditions $J=2$, $\Gamma=0.1 \mathrm{~s}^{-1}, \gamma=0.05 \mathrm{~s}^{-1}, \Gamma_{0}=0.01 \mathrm{~s}^{-1}, a=0.2, \delta=\pi / 3, \chi_{\mathrm{r}}=0.025, \omega=1 \mathrm{~s}^{-1}$ and initial phase $\chi=3 \pi / 2$.
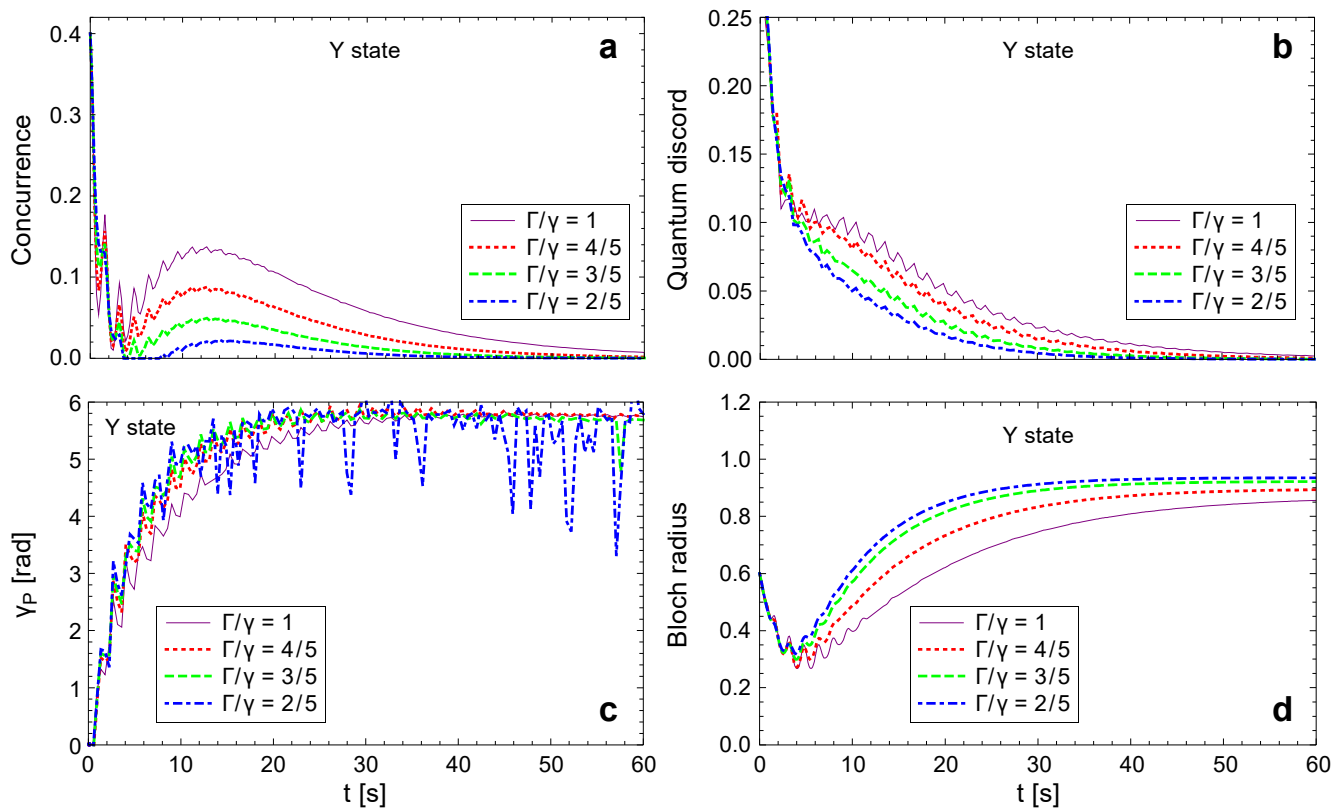

Fig. 7. Different ratios $\Gamma / \gamma$ of environments for concurrence (a), quantum discord (b), Pancharatnam phase (c), and Bloch radius (d) as a function of evolving time in the $Y$ state with the same initial conditions $J=2, \Gamma_{0}=0.01 \mathrm{~s}^{-1}, \gamma=0.05 \mathrm{~s}^{-1}, n=2, a=0.2, \delta=\pi / 3, \chi_{\mathrm{r}}=0.025, \omega=1 \mathrm{~s}^{-1}$ and initial phase $\chi=3 \pi / 2$. 

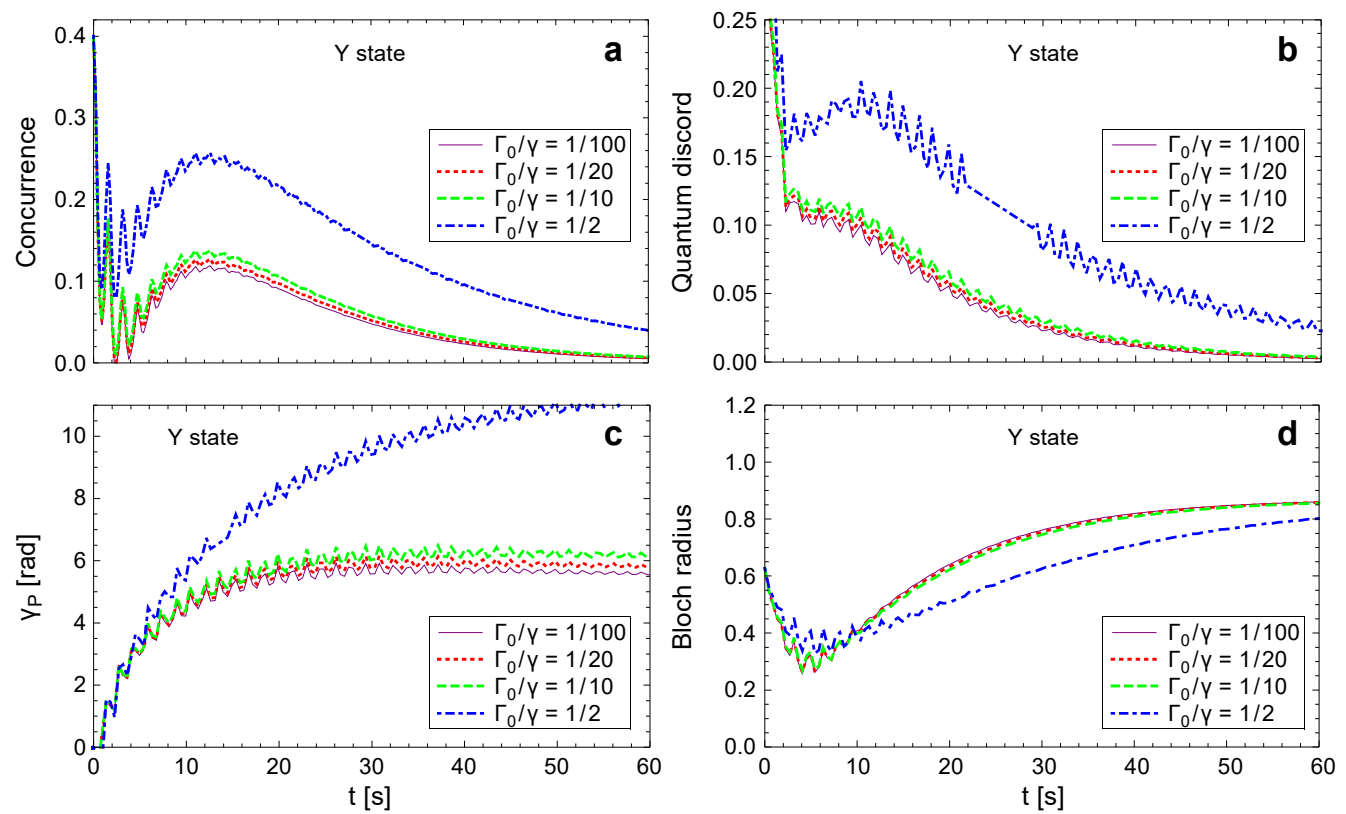

Fig. 8. Different ratios $\Gamma_{0} / \gamma$ of environments for concurrence (a), quantum discord (b), Pancharatnam phase (c), and Bloch radius (d) as a function of evolving time in the $Y$ state with the same initial conditions $J=2, \Gamma=0.1 \mathrm{~s}^{-1}, \gamma=0.05 \mathrm{~s}^{-1}, n=2, a=0.2, \delta=\pi / 3, \chi_{\mathrm{r}}=0.025, \omega=1 \mathrm{~s}^{-1}$ and initial phase $\chi=3 \pi / 2$.

For the $\mathrm{Y}$ state, the time-evolving curves of the quantum concurrence, discord, Pancharatnam phase and Bloch radius are shown in Figs. 6-8. Comparing Fig. 2 with Fig. 6, Fig. 4 with Fig. 7 and Fig. 5 with Fig. 8, we find that similar cases take place for the quantum concurrence and the discord. But the Pancharatnam phase is an increasing function of evolving time. Because the $\mathrm{X}$ and $\mathrm{Y}$ states have different non-diagonal elements, different coupling methods are needed. Therefore, the geometric phase provides a way how to distinguish the $\mathrm{X}$ and the $\mathrm{Y}$ states.

\section{Conclusions}

In summary, two coupling superconducting qubits are studied for their nonlocality, i.e., the quantum concurrence, discord, and Pancharatnam, for the $\mathrm{X}$ and $\mathrm{Y}$ states under all possible environments. We find that the $\mathrm{X}$ and $\mathrm{Y}$ states are special mixed states according to the Bloch radius, where the behavior of the Bloch radius for the two-particle system is different from the one of the single-particle system. Generally, the larger the environment and phonon number are at the larger region of time, the larger the quantum concurrence and discord are. Especially, the ratio of different environment correlations is important to implement the quantum computation. The Pancharatnam phases have different behaviors for the $\mathrm{X}$ and $\mathrm{Y}$ states.

Acknowledgments - This work was supported by the Jiangxi Education Department Fund (GJJ161060). 


\section{Appendix I}

According to SU(4) group, a set of $4 \times 4$ traceless and Hermitian matrices in Eq. (3) are given by:

$$
\begin{aligned}
& \lambda_{1}=\left(\begin{array}{cccc}
0 & 0 & 0 & 0 \\
0 & 0 & 1 & 0 \\
0 & 1 & 0 & 0 \\
0 & 0 & 0 & 0
\end{array}\right), \quad \lambda_{2}=\left(\begin{array}{cccc}
0 & 0 & 0 & 0 \\
0 & 0 & -i & 0 \\
0 & i & 0 & 0 \\
0 & 0 & 0 & 0
\end{array}\right), \quad \lambda_{3}=\left(\begin{array}{cccc}
0 & 0 & 0 & 0 \\
0 & 1 & 0 & 0 \\
0 & 0 & -1 & 0 \\
0 & 0 & 0 & 0
\end{array}\right), \quad \lambda_{4}=\left(\begin{array}{cccc}
0 & 0 & 0 & 0 \\
0 & 0 & 0 & 1 \\
0 & 0 & 0 & 0 \\
0 & 1 & 0 & 0
\end{array}\right), \\
& \lambda_{5}=\left(\begin{array}{cccc}
0 & 0 & 0 & 0 \\
0 & 0 & 0 & -i \\
0 & 0 & 0 & 0 \\
0 & i & 0 & 0
\end{array}\right), \quad \lambda_{6}=\left(\begin{array}{cccc}
0 & 0 & 0 & 0 \\
0 & 0 & 0 & 0 \\
0 & 0 & 0 & 1 \\
0 & 0 & 1 & 0
\end{array}\right), \quad \lambda_{7}=\left(\begin{array}{cccc}
0 & 0 & 0 & 0 \\
0 & 0 & 0 & 0 \\
0 & 0 & 0 & -i \\
0 & 0 & i & 0
\end{array}\right), \quad \lambda_{8}=\frac{1}{\sqrt{3}}\left(\begin{array}{cccc}
0 & 0 & 0 & 0 \\
0 & 1 & 0 & 0 \\
0 & 0 & 1 & 0 \\
0 & 0 & 0 & -2
\end{array}\right) \text {, } \\
& \lambda_{9}=\left(\begin{array}{llll}
0 & 1 & 0 & 0 \\
1 & 0 & 0 & 0 \\
0 & 0 & 0 & 0 \\
0 & 0 & 0 & 0
\end{array}\right), \quad \lambda_{10}=\left(\begin{array}{cccc}
0 & -i & 0 & 0 \\
i & 0 & 0 & 0 \\
0 & 0 & 0 & 0 \\
0 & 0 & 0 & 0
\end{array}\right), \quad \lambda_{11}=\left(\begin{array}{cccc}
0 & 0 & 1 & 0 \\
0 & 0 & 0 & 0 \\
1 & 0 & 0 & 0 \\
0 & 0 & 0 & 0
\end{array}\right), \quad \lambda_{12}=\left(\begin{array}{cccc}
0 & 0 & -i & 0 \\
0 & 0 & 0 & 0 \\
i & 0 & 0 & 0 \\
0 & 0 & 0 & 0
\end{array}\right), \\
& \lambda_{13}=\left(\begin{array}{cccc}
0 & 0 & 0 & 1 \\
0 & 0 & 0 & 0 \\
0 & 0 & 0 & 0 \\
1 & 0 & 0 & 0
\end{array}\right), \quad \lambda_{14}=\left(\begin{array}{cccc}
0 & 0 & 0 & -i \\
0 & 0 & 0 & 0 \\
0 & 0 & 0 & 0 \\
i & 0 & 0 & 0
\end{array}\right), \quad \lambda_{15}=\frac{1}{\sqrt{6}}\left(\begin{array}{cccc}
-3 & 0 & 0 & 0 \\
0 & 1 & 0 & 0 \\
0 & 0 & 1 & 0 \\
0 & 0 & 0 & 1
\end{array}\right)
\end{aligned}
$$

\section{Appendix II}

Calculated some parameter expressions in the density matrix in Section 4.

Parameters $X^{\prime \prime}, Y^{\prime \prime}, Z^{\prime \prime}, W^{\prime \prime}$ can be written as:

$$
\begin{aligned}
& X^{\prime \prime}=\frac{4(1-a) n^{2} \gamma \Gamma\left(4 J^{2}+n \gamma\left(n \gamma+2\left(-\Gamma+\Gamma_{0}\right)\right)\right)}{3 M_{1}} \\
& Y^{\prime \prime}=\frac{16 I(-1+a) n^{3} \gamma^{2} \Gamma \chi_{\mathrm{r}}}{3 M_{2}} \\
& Z^{\prime \prime}=\frac{\frac{2}{3}(-1+a)+4(1-a) n^{2} \Gamma^{2}\left(4 J^{2}+n \gamma\left(n \gamma+2\left(-\Gamma+\Gamma_{0}\right)\right)\right)}{3 M_{1}} \\
& W^{\prime \prime}=\frac{32(-1+a) J n^{2} \gamma \Gamma \chi_{\mathrm{r}}}{3 M_{2}}
\end{aligned}
$$

where

$$
\begin{aligned}
M_{1}= & n^{2} \Gamma^{2}\left(4 J^{2}+n \gamma\left(n \gamma+2\left(-\Gamma+\Gamma_{0}\right)\right)\right) \\
& +n \gamma\left(-\left(n \gamma+2\left(-\Gamma+\Gamma_{0}\right)\right)\left(4 J^{2}+n \gamma\left(n \gamma+2\left(-\Gamma+\Gamma_{0}\right)\right)\right)-16 n^{3} \gamma \chi_{\mathrm{r}}^{2}\right) \\
M_{2}= & 4 J^{2} n \gamma^{2}+n^{3} \gamma^{4}-8 J^{2} \gamma \Gamma-4 n^{2} \gamma^{3} \Gamma-4 J^{2} n \Gamma^{2}+4 n \gamma^{2} \Gamma^{2}-n^{3} \gamma^{2} \Gamma^{2}+2 n^{2} \gamma \Gamma^{3} \\
& +8 J^{2} \gamma \Gamma_{0}+4 n^{2} \gamma^{3} \Gamma_{0}-8 n \gamma^{2} \Gamma \Gamma_{0}-2 n^{2} \gamma \Gamma^{2} \Gamma_{0}+4 n \gamma^{2} \Gamma_{0}^{2}+16 n^{3} \gamma^{2} \chi_{\mathrm{r}}^{2}
\end{aligned}
$$


Time-dependent Pancharatnam phases and quantum correlations...

Parameters $x_{1}, x_{2}, x_{3}$, and $x_{i}$ can be written as:

$$
\begin{aligned}
x_{1} & =-\frac{m_{4}}{4}-\frac{1}{2} \sqrt{-\frac{2 m_{3}}{3}+\frac{m_{4}^{2}}{4}+\frac{2^{1 / 3} m_{5}}{3 m_{7}}+\frac{m_{7}}{3 \times 2^{1 / 3}}} \\
- & \sqrt{-\frac{4 m_{3}}{3}+\frac{m_{4}^{2}}{2}-\frac{2^{1 / 3} m_{5}}{3 m_{7}}-\frac{m_{7}}{3 \times 2^{1 / 3}}-\frac{-8 m_{2}+4 m_{3} m_{4}-m_{4}^{3}}{4\left(-\frac{2 m_{3}}{3}+\frac{m_{4}^{2}}{4}+\frac{2^{1 / 3} m_{5}}{3 m_{7}}+\frac{m_{7}}{3 \times 2^{1 / 3}}\right)^{1 / 2}}} \\
x_{2} & =-\frac{m_{4}}{4}-\frac{1}{2} \sqrt{-\frac{2 m_{3}}{3}+\frac{m_{4}^{2}}{4}+\frac{2^{1 / 3} m_{5}}{3 m_{7}}+\frac{m_{7}}{3 \times 2^{1 / 3}}} \\
& +\frac{1}{-\frac{4 m_{3}}{3}+\frac{m_{4}^{2}}{2}-\frac{2^{1 / 3} m_{5}}{3 m_{7}}-\frac{m_{7}}{3 \times 2^{1 / 3}}-\frac{4\left(-\frac{2 m_{3}}{3}+\frac{m_{4}^{2}}{4}+\frac{2^{1 / 3} m_{5}}{3 m_{7}}+\frac{m_{7}}{3 \times 2^{1 / 3}}\right)^{1 / 2}}{3}} \\
x_{\mathrm{r}} & =-\frac{m_{4}}{4}+\frac{1}{2} \sqrt{-\frac{2 m_{3}}{3}+\frac{m_{4}^{2}}{4}+\frac{2^{1 / 3} m_{5}}{3 m_{7}}+\frac{m_{7}}{3 \times 2^{1 / 3}}} \\
x_{\mathrm{i}} & =\frac{i}{2} \sqrt{-\frac{m_{3}}{3}+\frac{m_{4}^{2}}{2}-\frac{2^{1 / 3} m_{5}}{3 m_{7}}-\frac{m_{7}}{3 \times 2^{1 / 3}}+\frac{m_{4}^{3}}{4\left(-\frac{2 m_{3}}{3}+\frac{m_{4}^{2}}{4}+\frac{2^{1 / 3} m_{5}}{3 m_{7}}+\frac{m_{7}}{3 \times 2^{1 / 3}}\right)^{1 / 2}}}
\end{aligned}
$$

where

$$
\begin{aligned}
m_{1}= & 4 J^{2} n^{2} \gamma^{2}+n^{4} \gamma^{4}-8 J^{2} n \gamma \Gamma-4 J^{2} n^{2} \Gamma^{2}-4 n^{2} \gamma^{2} \Gamma^{2}-n^{4} \gamma^{2} \Gamma^{2}-2 n^{3} \gamma \Gamma^{3} \\
& +8 J^{2} n \gamma \Gamma_{0}+4 n^{3} \gamma^{3} \Gamma_{0}-2 n^{3} \gamma \Gamma^{2} \Gamma_{0}+4 n^{2} \gamma^{2} \Gamma_{0}^{2}+16 n^{4} \gamma^{2} \chi_{\mathrm{r}}^{2} \\
m_{2}= & -8 J^{2} n \gamma-4 n^{3} \gamma^{3}+8 J^{2} \Gamma+8 n \gamma \Gamma^{2}+2 n^{3} \gamma \Gamma^{2}+2 n^{2} \Gamma^{3}-8 J^{2} \Gamma_{0}-12 n^{2} \gamma^{2} \Gamma_{0} \\
& +2 n^{2} \Gamma^{2} \Gamma_{0}-8 n \gamma \Gamma_{0}^{2}-32 n^{3} \gamma \chi_{\mathrm{r}}^{2} \\
m_{3}= & 4 J^{2}+6 n^{2} \gamma^{2}-4 \Gamma^{2}-n^{2} \Gamma^{2}+12 n \gamma \Gamma_{0}+4 \Gamma_{0}^{2}+16 n^{2} \chi_{\mathrm{r}}^{2} \\
m_{4}= & -4 n \gamma-4 \Gamma_{0} \\
m_{5}= & 12 m_{1}+m_{3}^{2}-3 m_{2} m_{4} \\
m_{6}= & 27 m_{2}^{2}-72 m_{1} m_{3}+2 m_{3}^{3}-9 m_{2} m_{3} m_{4}+27 m_{1} m_{4}^{2} \\
m_{7}= & m_{6}+\left(-4 m_{5}^{3}+m_{6}^{2}\right)^{1 / 3}
\end{aligned}
$$

Parameters $h_{1}, h_{2}, h_{3}, h_{4}, h_{\mathrm{r}}, h_{\mathrm{i}}$, and $\beta_{\mathrm{r}}, \beta_{\mathrm{i}}$ can be written as:

$$
h_{1}=\frac{1}{8 J n^{2} \Gamma \chi_{\mathrm{r}}}\left(4 J^{2} n \Gamma+n \Gamma\left(n \gamma-x_{1}\right)\left(n \gamma+2\left(\gamma+\Gamma_{0}\right)-x_{1}\right)\right)
$$




$$
\begin{aligned}
& h_{2}=\frac{1}{8 J n \chi_{\mathrm{r}}\left(n \gamma-x_{1}\right)}\left(4 J^{2} n \Gamma+n \Gamma\left(n \gamma-x_{1}\right)\left(n \gamma+2\left(\Gamma+\Gamma_{0}\right)-x_{1}\right)\right) \\
& h_{3}=\frac{1}{8 J n^{2} \Gamma \chi_{\mathrm{r}}}\left(4 J^{2} n \Gamma+n \Gamma\left(n \gamma-x_{2}\right)\left(n \gamma+2\left(\gamma+\Gamma_{0}\right)-x_{2}\right)\right) \\
& h_{4}=\frac{1}{8 J n \chi_{\mathrm{r}}\left(n \gamma-x_{2}\right)}\left(4 J^{2} n \Gamma+n \Gamma\left(n \gamma-x_{2}\right)\left(n \gamma+2\left(\Gamma+\Gamma_{0}\right)-x_{2}\right)\right) \\
& h_{\mathrm{r}}=\frac{1}{8 J n \chi_{\mathrm{r}}}\left(4 J^{2}-x_{\mathrm{i}}^{2}+\left(n \gamma-x_{\mathrm{r}}\right)\left(n \gamma+2\left(\Gamma+\Gamma_{0}\right)-x_{\mathrm{r}}\right)\right) \\
& h_{\mathrm{i}}=-\frac{1}{4 J n \chi_{\mathrm{r}}}\left(x_{\mathrm{i}}\left(n \gamma+\Gamma+\Gamma_{0}-x_{\mathrm{r}}\right)\right) \\
& \beta_{\mathrm{r}}=\frac{J \Gamma\left(n \gamma-x_{\mathrm{r}}\right)}{2 x_{\mathrm{r}}\left(n \gamma-x_{\mathrm{r}}\right)^{2}+x_{\mathrm{i}}^{2}}+\frac{n \Gamma\left(n \gamma+2\left(\Gamma+\Gamma_{0}\right)-x_{\mathrm{r}}\right)}{8 J n x_{\mathrm{r}}} \\
& \beta_{\mathrm{i}}=\frac{x_{\mathrm{i}} J \Gamma}{2 x_{\mathrm{r}}\left(n \gamma-x_{\mathrm{r}}\right)^{2}+x_{\mathrm{i}}^{2}}-\frac{x_{\mathrm{i}} n \Gamma}{8 J n x_{\mathrm{r}}}
\end{aligned}
$$

and

$$
\begin{aligned}
& c_{1}=\left[6 i c_{\mathrm{y}} h_{4} h_{\mathrm{i}} J+2 h_{\mathrm{i}} x_{2}-3 c_{\mathrm{z}} h_{\mathrm{i}} x_{2}-2 h_{3} x_{\mathrm{i}}+3 c_{\mathrm{z}} h_{3} x_{\mathrm{i}}-3 c_{\mathrm{x}} h_{4} x_{\mathrm{i}}+2 h_{\mathrm{r}} x_{\mathrm{i}}-3 c_{\mathrm{z}} h_{\mathrm{r}} x_{\mathrm{i}}\right. \\
& +3 c_{\mathrm{w}} h_{4} h_{\mathrm{r}} x_{\mathrm{i}}-2 h_{\mathrm{i}} x_{\mathrm{r}}+3 c_{\mathrm{z}} h_{\mathrm{i}} x_{\mathrm{r}}-3 c_{\mathrm{w}} h_{4} h_{\mathrm{i}} x_{\mathrm{r}}-6 i c_{\mathrm{y}} h_{3} J \beta_{\mathrm{i}}+6 i c_{\mathrm{y}} h_{\mathrm{r}} J \beta_{\mathrm{i}} \\
& +3 c_{\mathrm{x}} x_{2} \beta_{\mathrm{i}}-3 c_{\mathrm{w}} h_{\mathrm{r}} x_{2} \beta_{\mathrm{i}}-3 c_{\mathrm{x}} x_{\mathrm{r}} \beta_{\mathrm{i}}+3 c_{\mathrm{w}} h_{3} x_{\mathrm{r}} \beta_{\mathrm{i}}-6 i c_{\mathrm{y}} h_{\mathrm{i}} J \beta_{\mathrm{r}}+3 c_{\mathrm{w}} h_{\mathrm{i}} x_{2} \beta_{\mathrm{r}}+3 c_{\mathrm{x}} x_{\mathrm{i}} \beta_{\mathrm{r}} \\
& -3 c_{\mathrm{w}} h_{3} x_{\mathrm{i}} \beta_{\mathrm{r}}+3 c_{\mathrm{w}} h_{4} h_{\mathrm{i}} n \gamma-3 c_{\mathrm{w}} h_{3} n \beta_{\mathrm{i}} \gamma+3 c_{\mathrm{w}} h_{\mathrm{r}} n \beta_{\mathrm{i}} \gamma-3 c_{\mathrm{w}} h_{\mathrm{i}} n \beta_{\mathrm{r}} \gamma \\
& \left.+2\left(h_{4} x_{\mathrm{i}}-x_{2} \beta_{\mathrm{i}}+x_{\mathrm{r}} \beta_{\mathrm{i}}-x_{\mathrm{i}} \beta_{\mathrm{r}}\right) \cos \chi+4 J\left(h_{4} h_{\mathrm{i}}-h_{3} \beta_{\mathrm{i}}+h_{\mathrm{r}} \beta_{\mathrm{i}}-h_{\mathrm{i}} \beta_{\mathrm{r}}\right) \sin \chi\right] \\
& \times\left[3 \left(h_{2}\left(h_{\mathrm{i}} x_{2}-h_{3} x_{\mathrm{i}}+h_{\mathrm{r}} x_{\mathrm{i}}-h_{\mathrm{i}} x_{\mathrm{r}}\right)+h_{4}\left(\left(h_{1}-h_{\mathrm{r}}\right) x_{\mathrm{i}}+h_{\mathrm{i}}\left(-x_{1}+x_{\mathrm{r}}\right)\right)\right.\right. \\
& \left.\left.+h_{3} x_{1} \beta_{\mathrm{i}}-h_{\mathrm{r}} x_{1} \beta_{\mathrm{i}}-h_{1} x_{2} \beta_{\mathrm{i}}+h_{\mathrm{r}} x_{2} \beta_{\mathrm{i}}+h_{1} x_{\mathrm{r}} \beta_{\mathrm{i}}-h_{3} x_{\mathrm{r}} \beta_{\mathrm{i}}+h_{\mathrm{i}} x_{1} \beta_{\mathrm{r}}-h_{\mathrm{i}} x_{2} \beta_{\mathrm{r}}-h_{1} x_{\mathrm{i}} \beta_{\mathrm{r}}+h_{3} x_{\mathrm{i}} \beta_{\mathrm{r}}\right)\right]^{-1} \\
& c_{2}=\left[6 i c_{\mathrm{y}} h_{2} h_{\mathrm{i}} J+2 h_{\mathrm{i}} x_{1}-3 c_{\mathrm{z}} h_{\mathrm{i}} x_{1}-2 h_{1} x_{\mathrm{i}}+3 c_{\mathrm{z}} h_{1} x_{\mathrm{i}}-3 c_{\mathrm{x}} h_{2} x_{\mathrm{i}}+2 h_{\mathrm{r}} x_{\mathrm{i}}-3 c_{\mathrm{z}} h_{\mathrm{r}} x_{\mathrm{i}}\right. \\
& +3 c_{\mathrm{w}} h_{2} h_{\mathrm{r}} x_{\mathrm{i}}-2 h_{\mathrm{i}} x_{\mathrm{r}}+3 c_{\mathrm{z}} h_{\mathrm{i}} x_{\mathrm{r}}-3 c_{\mathrm{w}} h_{2} h_{\mathrm{i}} x_{\mathrm{r}}-6 i c_{\mathrm{y}} h_{1} J \beta_{\mathrm{i}}+6 i c_{\mathrm{y}} h_{\mathrm{r}} J \beta_{\mathrm{i}} \\
& +3 c_{\mathrm{x}} x_{1} \beta_{\mathrm{i}}-3 c_{\mathrm{w}} h_{\mathrm{r}} x_{1} \beta_{\mathrm{i}}-3 c_{\mathrm{x}} x_{\mathrm{r}} \beta_{\mathrm{i}}+3 c_{\mathrm{w}} h_{1} x_{\mathrm{r}} \beta_{\mathrm{i}}-6 i c_{\mathrm{y}} h_{\mathrm{i}} J \beta_{\mathrm{r}}+3 c_{\mathrm{w}} h_{\mathrm{i}} x_{1} \beta_{\mathrm{r}}+3 c_{\mathrm{x}} x_{\mathrm{i}} \beta_{\mathrm{r}} \\
& -3 c_{\mathrm{w}} h_{1} x_{\mathrm{i}} \beta_{\mathrm{r}}+3 c_{\mathrm{w}} h_{2} h_{\mathrm{i}} n \gamma-3 c_{\mathrm{w}} h_{1} n \beta_{\mathrm{i}} \gamma+3 c_{\mathrm{w}} h_{\mathrm{r}} n \beta_{\mathrm{i}} \gamma-3 c_{\mathrm{w}} h_{\mathrm{i}} n \beta_{\mathrm{r}} \gamma \\
& \left.+2\left(h_{2} x_{\mathrm{i}}-x_{\mathrm{i}} \beta_{\mathrm{i}}+x_{\mathrm{r}} \beta_{\mathrm{i}}-x_{\mathrm{i}} \beta_{\mathrm{r}}\right) \cos \chi+4 J\left(h_{2} h_{\mathrm{i}}-h_{1} \beta_{\mathrm{i}}+h_{\mathrm{r}} \beta_{\mathrm{i}}-h_{\mathrm{i}} \beta_{\mathrm{r}}\right) \sin \chi\right] \\
& \times\left[3 \left(h_{4}\left(\left(-h_{1}+h_{\mathrm{r}}\right) x_{\mathrm{i}}+h_{\mathrm{i}}\left(x_{1}-x_{\mathrm{r}}\right)\right)+h_{2}\left(-h_{\mathrm{i}} x_{2}+h_{3} x_{\mathrm{i}}-h_{\mathrm{r}} x_{\mathrm{i}}+h_{\mathrm{i}} x_{\mathrm{r}}\right)\right.\right. \\
& \left.\left.-h_{3} x_{1} \beta_{\mathrm{i}}+h_{\mathrm{r}} x_{1} \beta_{\mathrm{i}}+h_{1} x_{2} \beta_{\mathrm{i}}-h_{\mathrm{r}} x_{2} \beta_{\mathrm{i}}-h_{1} x_{\mathrm{r}} \beta_{\mathrm{i}}+h_{3} x_{\mathrm{r}} \beta_{\mathrm{i}}-h_{\mathrm{i}} x_{1} \beta_{\mathrm{r}}+h_{\mathrm{i}} x_{2} \beta_{\mathrm{r}}+h_{1} x_{\mathrm{i}} \beta_{\mathrm{r}}-h_{3} x_{\mathrm{i}} \beta_{\mathrm{r}}\right)\right]^{-1} \\
& c_{\mathrm{r}}=\left[6 i c_{\mathrm{y}} h_{2} h_{\mathrm{i}} J-6 i c_{\mathrm{y}} h_{4} h_{\mathrm{i}} J+2 h_{\mathrm{i}} x_{1}-3 c_{\mathrm{z}} h_{\mathrm{i}} x_{1}+3 c_{\mathrm{w}} h_{3} h_{\mathrm{i}} x_{1}-2 h_{\mathrm{i}} x_{2}+3 c_{\mathrm{z}} h_{\mathrm{i}} x_{2}-3 c_{\mathrm{w}} h_{2} h_{\mathrm{i}} x_{2}\right. \\
& -2 h_{1} x_{\mathrm{i}}+3 c_{\mathrm{z}} h_{1} x_{\mathrm{i}}-3 c_{\mathrm{x}} h_{2} x_{\mathrm{i}}+2 h_{3} x_{\mathrm{i}}-3 c_{\mathrm{z}} h_{3} x_{\mathrm{i}}+3 c_{\mathrm{w}} h_{2} h_{3} x_{\mathrm{i}}+3 c_{\mathrm{x}} h_{4} x_{\mathrm{i}}-3 c_{\mathrm{w}} h_{1} h_{4} x_{\mathrm{i}} \\
& -6 i c_{\mathrm{y}} h_{1} J \beta_{\mathrm{i}}+6 i c_{\mathrm{y}} h_{3} J \beta_{\mathrm{i}}+3 c_{\mathrm{x}} x_{1} \beta_{\mathrm{i}}-3 c_{\mathrm{w}} h_{3} x_{1} \beta_{\mathrm{i}}-3 c_{\mathrm{x}} x_{2} \beta_{\mathrm{i}}+3 c_{\mathrm{w}} h_{1} x_{2} \beta_{\mathrm{i}} \\
& +3 c_{\mathrm{w}} h_{3} h_{\mathrm{i}} n \gamma-3 c_{\mathrm{w}} h_{4} h_{\mathrm{i}} n \gamma-3 c_{\mathrm{w}} h_{1} n \beta_{\mathrm{i}} \gamma+3 c_{\mathrm{w}} h_{3} n \beta_{\mathrm{i}} \gamma \\
& \left.+2\left(h_{2} x_{\mathrm{i}}-h_{4} x_{\mathrm{i}}-x_{1} \beta_{\mathrm{i}}+x_{2} \beta_{\mathrm{i}}\right) \cos \chi+4 J\left(h_{2} h_{\mathrm{i}}-h_{4} h_{\mathrm{i}}-h_{1} \beta_{\mathrm{i}}+h_{3} \beta_{\mathrm{r}}\right) \sin \chi\right] \\
& \times\left[6 \left(h_{2}\left(h_{\mathrm{i}} x_{2}-h_{3} x_{\mathrm{i}}+h_{\mathrm{r}} x_{\mathrm{i}}-h_{\mathrm{i}} x_{\mathrm{r}}\right)+h_{4}\left(\left(h_{1}-h_{\mathrm{r}}\right) x_{\mathrm{i}}+h_{\mathrm{i}}\left(-x_{1}+x_{\mathrm{r}}\right)\right)\right.\right. \\
& \left.\left.+h_{3} x_{1} \beta_{\mathrm{i}}-h_{\mathrm{r}} x_{1} \beta_{\mathrm{i}}-h_{1} x_{2} \beta_{\mathrm{i}}+h_{\mathrm{r}} x_{2} \beta_{\mathrm{i}}+h_{1} x_{\mathrm{r}} \beta_{\mathrm{i}}-h_{3} x_{\mathrm{r}} \beta_{\mathrm{i}}+h_{\mathrm{i}} x_{1} \beta_{\mathrm{r}}-h_{\mathrm{i}} x_{2} \beta_{\mathrm{r}}-h_{1} x_{\mathrm{i}} \beta_{\mathrm{r}}+h_{3} x_{\mathrm{i}} \beta_{\mathrm{r}}\right)\right]^{-1}
\end{aligned}
$$




$$
\begin{aligned}
c_{\mathrm{i}}= & {\left[6 i c_{\mathrm{y}} h_{2} h_{3} J-6 i c_{\mathrm{y}} h_{1} h_{4} J-6 i c_{\mathrm{y}} h_{2} h_{\mathrm{r}} J+6 i c_{\mathrm{y}} h_{4} h_{\mathrm{r}} J+2 h_{3} x_{1}-3 c_{\mathrm{z}} h_{3} x_{1}+3 c_{\mathrm{x}} h_{4} x_{1}\right.} \\
& -2 h_{\mathrm{r}} x_{1}+3 c_{\mathrm{z}} h_{\mathrm{r}} x_{1}-3 c_{\mathrm{w}} h_{4} x_{1}-2 h_{1} x_{2}+3 c_{\mathrm{z}} h_{1} x_{2}-3 c_{\mathrm{x}} h_{2} x_{2}+2 h_{\mathrm{r}} x_{2}-3 c_{\mathrm{z}} h_{\mathrm{r}} x_{2} \\
& +3 c_{\mathrm{w}} h_{2} h_{\mathrm{r}} x_{2}+2 h_{1} x_{\mathrm{r}}-3 c_{\mathrm{z}} h_{1} x_{\mathrm{r}}+3 c_{\mathrm{x}} h_{2} x_{\mathrm{r}}-2 h_{3} x_{\mathrm{r}}+3 c_{\mathrm{z}} h_{3} x_{\mathrm{r}} \\
& -3 c_{\mathrm{w}} h_{2} h_{4} x_{\mathrm{r}}-3 c_{\mathrm{x}} h_{4} x_{\mathrm{r}}+3 c_{\mathrm{w}} h_{1} h_{4} x_{\mathrm{r}}+6 i c_{\mathrm{y}} h_{1} J \beta_{\mathrm{r}}-6 i c_{\mathrm{y}} h_{3} J \beta_{\mathrm{r}}-3 c_{\mathrm{x}} x_{1} \beta_{\mathrm{r}} \\
& +3 c_{\mathrm{w}} h_{3} x_{1} \beta_{\mathrm{r}}+3 c_{\mathrm{x}} x_{2} \beta_{\mathrm{r}}-3 c_{\mathrm{w}} h_{1} x_{2} \beta_{\mathrm{r}}+3 c_{\mathrm{w}} h_{2} h_{3} n \gamma-3 c_{\mathrm{w}} h_{1} h_{4} n \gamma \\
& -3 c_{\mathrm{w}} h_{2} h_{\mathrm{r}} n \gamma+3 c_{\mathrm{w}} h_{4} h_{\mathrm{r}} n \gamma+3 c_{\mathrm{w}} h_{1} n \beta_{\mathrm{r}} \gamma-3 c_{\mathrm{w}} h_{3} n \beta_{\mathrm{r}} \gamma \\
& +2\left(h_{2} x_{2}-h_{2} x_{\mathrm{r}}+h_{4}\left(-x_{1}+x_{\mathrm{r}}\right)+x_{1} \beta_{\mathrm{r}}-x_{2} \beta_{\mathrm{r}}\right) \cos \chi \\
& \left.+4 J\left(h_{2} h_{3}-h_{1} h_{4}-h_{2} h_{\mathrm{r}}+h_{4} h_{\mathrm{r}}+h_{1} \beta_{\mathrm{r}}-h_{3} \beta_{\mathrm{r}}\right) \sin \chi\right] \\
& \times\left[6 \left(h_{4}\left(\left(-h_{1}+h_{\mathrm{r}}\right) x_{\mathrm{i}}+h_{\mathrm{i}}\left(x_{1}-x_{\mathrm{r}}\right)\right)+h_{2}\left(-h_{\mathrm{i}} x_{2}+h_{3} x_{\mathrm{i}}-h_{\mathrm{r}} x_{\mathrm{i}}+h_{\mathrm{i}} x_{\mathrm{r}}\right)\right.\right. \\
& \left.\left.-h_{3} x_{1} \beta_{\mathrm{i}}+h_{\mathrm{r}} \beta_{\mathrm{i}}+h_{1} x_{2} \beta_{\mathrm{i}}-h_{\mathrm{r}} x_{2} \beta_{\mathrm{i}}-h_{1} x_{\mathrm{r}} \beta_{\mathrm{i}}+h_{3} x_{\mathrm{r}} \beta_{\mathrm{i}}-h_{\mathrm{i}} x_{1} \beta_{\mathrm{r}}+h_{\mathrm{i}} x_{2} \beta_{\mathrm{r}}+h_{1} x_{\mathrm{i}} \beta_{\mathrm{r}}-h_{3} x_{\mathrm{i}} \beta_{\mathrm{r}}\right)\right]^{-1}
\end{aligned}
$$

\section{References}

[1] Cirac J.I., Zoller P., Quantum computations with cold trapped ions, Physical Review Letters 74(20), 1995, pp. 4091-4094, DOI: 10.1103/PhysRevLett.74.4091; CiraC J.I., Zoller P., A scalable quantum computer with ions in an array of microtraps, Nature 404, 2000, pp. 579-581, DOI: $10.1038 / 35007021$.

[2] Wootters W.K., Entanglement of formation of an arbitrary state of two qubits, Physical Review Letters 80(10), 1998, pp. 2245-2248, DOI: 10.1103/PhysRevLett.80.2245.

[3] Henderson L., Vedral V., Classical, quantum and total correlations, Journal of Physics A: Mathematical and General 34(35), 2001, pp. 6899-6905, DOI: 10.1088/0305-4470/34/35/315.

[4] Ollivier H., ZuReK W.H., Quantum discord: a measure of the quantumness of correlations, Physical Review Letters 88(1), 2002, article 017901, DOI: 10.1103/PhysRevLett.88.017901.

[5] WANG Z.S., Geometric quantum computation and dynamical invariant operators, Physical Review A 79(2), 2009, article 024304, DOI: 10.1103/PhysRevA.79.024304.

[6] Wang Z.S., LiU G.Q., Ji Y.H., Noncyclic geometric quantum computation in a nuclear-magneticresonance system, Physical Review A 79(5), 2009, article 054301, DOI: 10.1103/PhysRevA.79.054301.

[7] Wang Z.S., Wu C., Feng X.-L., Kwek L.C., Lai C.H., OH C.H., Vedral V., Nonadiabatic geometric quantum computation, Physical Review A 76(4), 2007, article 044303, DOI: 10.1103/PhysRevA.76. $\underline{044303}$.

[8] Troiani F., Hohenester U., Molinari U., Exploiting exciton-exciton interactions in semiconductor quantum dots for quantum-information processing, Physical Review B 62(4), 2000, pp. R2263-R2266, DOI: 10.1103/PhysRevB.62.R2263.

[9] Biolatti E., IotTi R.C., Zanardi P., Rossi F., Quantum information processing with semiconductor macroatoms, Physical Review Letters 85(26), 2000, pp. 5647-5650, DOI: 10.1103/PhysRevLett.85.5647.

[10] Flindt C., Sørensen A.S., Lukin M.D., Taylor J.M., Spin-photon entangling diode, Physical Review Letters 98(24), 2007, article 240501, DOI: 10.1103/PhysRevLett.98.240501.

[11] Corrielli G., Crespi A., Geremia R., Ramponi R., Sansoni L., Santinelli A., Mataloni P., Sciarrino F., Osellame R., Rotated waveplates in integrated waveguide optics, Nature Communications 5, 2014, article 4249, DOI: $10.1038 /$ ncomms5249.

[12] Pires D.P., Silva I.A., DeAzevedo E.R., Soares-Pinto D.O., Filgueiras J.G., Coherence orders, decoherence, and quantum metrology, Physical Review A 98(3), 2018, article 032101, DOI: 10.1103 / PhysRevA.98.032101.

[13] Yu Y.X., Fu G.L., GuO L.P., PAn H., Wang Z.S., Quantum correlations of coupled superconducting two-qubit system in various cavity environments, Physica C 495, 2013, pp. 88-108, DOI: $10.1016 /$ j.physc.2013.08.007.

[14] Fu G.L., Chen Z.Q., Rao H.Y., Guo L.P., Wang Z.S., Fidelity and entanglement with environment effect, International Journal of Theoretical Physics 53(1), 2014, pp. 146-158, DOI: 10.1007/s10773 -013-1792-Z. 
[15] Huang Y.-Y., Wu Y.-K., Wang F., Hou P.-Y., Wang W.-B., Zhang W.-G., Lian W.-Q., Liu Y.-Q., Wang H.-Y., Zhang H.-Y., He L., Chang X.-Y., Xu Y., Duan L.-M., Experimental realization of robust geometric quantum gates with solid-state spins, Physical Review Letters 122(1), 2019, article 010503, DOI: 10.1103/PhysRevLett.122.010503.

[16] Chiorescu I., Nakamura Y., Harmans C.J.P.M., Mooij J.E., Coherent quantum dynamics of a superconducting flux qubit, Science 299(5614), 2003, pp. 1869-1871, DOI: 10.1126/science. 1081045.

[17] Viola L., Knill E., Lloyd S., Dynamical decoupling of open quantum systems, Physical Review Letters 82(12), 1999, pp. 2417-2421, DOI: 10.1103/PhysRevLett.82.2417.

[18] Wang Z.S., LiU Q., Geometric phase and spinorial representation of mixed state, Physics Letters A 377(45-48), 2013, pp. 3272-3278, DOI: 10.1016/j.physleta.2013.10.023.

[19] Xiao D., Chang M.C., Niu Q., Berry phase effects on electronic properties, Reviews of Modern Physics 82(3), 2010, pp. 1959-2007, DOI: 10.1103/RevModPhys.82.1959.

[20] WANG Z.S., PAN H., Geometric phase carried by the observables and its application to quantum computation, Quantum Information and Computation 15(11-12), 2015, pp. 951-961.

[21] Falci G., Fazio R., Massimo Palma G., Siewert J., Vedral V., Detection of geometric phases in superconducting nanocircuits, Nature 407, 2000, pp. 355-358, DOI: 10.1038/35030052.

[22] Ekert A., Ericsson M., Hayden P., Inamori H., Jones J.A., Oi D.K.L., Vedral V., Geometric quantum computation, Journal of Modern Optics 47(14-15), 2000, pp. 2501-2513.

[23] Steffen M., Ansmann M., Bialczak R.C., Katz N., Lucero E., McDermott R., Neeley M., Weig E.M., Cleland A.N., Martinis J.M., Measurement of the entanglement of two superconducting qubits via state tomography, Science 313(5792), 2006, pp. 1423-1425, DOI: 10.1126/science.1130886.

[24] TANG L., LIU F., Generation of multipartite entangled coherent states via a superconducting charge qubit, Physics Letters A 378(30-31), pp. 2014-2078, 2074, DOI: 10.1016/j.physleta.2014.05.054.

[25] Kiktenko E.O., Fedorov A.K., Strakhov A.A., Man'ko V.I., Single qudit realization of the Deutsch algorithm using superconducting many-level quantum circuits, Physics Letters A 379(22-23), 2015, pp. 1409-1413, DOI: $10.1016 /$ j.physleta.2015.03.023.

[26] Nunes L.H.C.M., Farias R.L.S., Marino E.C., Superconducting and excitonic quantum phase transitions in doped Dirac electronic systems, Physics Letters A 376(5), 2012, pp. 779-784, DOI: 10.1016/ j.physleta.2011.12.030.

[27] Y ABU-UTI B.F.C., Roversi J.A., Implementation of a two-qubit controlled-rotation gate based on unconventional geometric phase with a constant gating time, Physics Letters A 375(36), 2011, pp. 3171 -3175, DOI: $10.1016 /$ j.physleta.2011.07.016.

[28] Он S., Geometric phases and entanglement of two qubits with XY type interaction, Physics Letters A 373(6), 2009, pp. 644-647, DOI: 10.1016/j.physleta.2008.12.023.

[29] Kiktenko E., Fedorov A., Tomographic causal analysis of two-qubit states and tomographic discord, Physics Letters A 378(24-25), 2014, pp. 1704-1710, DOI: 10.1016/j.physleta.2014.04.036.

[30] Konyk W., Gea-Banacloche J., Passive, deterministic photonic conditional-phase gate via two-level systems, Physical Review A 99(1), 2019, article 010301(R), DOI: 10.1103/PhysRevA.99.010301.

[31] JAFARI R., Quantum renormalization group approach to geometric phases in spin chains, Physics Letters A 377(45-48), 2013, pp. 3279-3282, DOI: 10.1016/j.physleta.2013.10.034.

[32] Zhang X.X., Zhang A.P., Li F.L., Detecting the multi-spin interaction of an XY spin chain by the geometric phase of a coupled qubit, Physics Letters A 376(30-31), 2012, pp. 2090-2095, DOI: 10.1016/j.physleta.2012.05.018.

[33] Gong L.Y., Zhu H., Zhao S.M., Cheng W.W., Sheng Y.B., Quantum discord and classical correlation signatures of mobility edges in one-dimensional aperiodic single-electron systems, Physics Letters A 376(45), 2012, pp. 3026-3032, DOI: 10.1016/j.physleta.2012.09.049.

[34] Girolami D., How difficult is it to prepare a quantum state?, Physical Review Letters 122(1), 2019, article 010505, DOI: 10.1103/PhysRevLett.122.010505.

[35] Blais A., Gambetta J., Wallraff A., Schuster D.I., Girvin S.M., Devoret M.H., Schoelkopf R.J., Quantum-information processing with circuit quantum electrodynamics, Physical Review A 75(3), 2007, article 032329, DOI: 10.1103/PhysRevA.75.032329. 
[36] Blais A., Huang R.-S., Wallraff A., Girvin S.M., Schoelkopf R.J., Cavity quantum electrodynamics for superconducting electrical circuits: An architecture for quantum computation, Physical Review A 69(6), 2004, article 062320, DOI: 10.1103/PhysRevA.69.062320.

[37] Majer J., Chow J.M., Gambetta J.M., Koch J., Johnson B.R., Schreier J.A., Frunzio L., Schuster D.I., Houck A.A., Wallraff A., Blais A., Devoret M.H., Girvin S.M., Schoelkopf R.J., Coupling superconducting qubits via a cavity bus, Nature 449, 2007, pp. 443-447, DOI: 10.1038/nature06184.

[38] LindBlad G., On the generators of quantum dynamical semigroups, Communications in Mathematical Physics 48, 1976, pp. 119-130, DOI: 10.1007/BF01608499.

[39] XIE B., ZENG G.R., PAN H., WANG Z.S., Pancharatnam phase and quantum correlation for two-qubit system in correlated dephasing environment, International Journal of Theoretical Physics 55, 2016, pp. 1474-1491, DOI: 10.1007/s10773-015-2786-9.

[40] Xue L.Y., Yu Y.X., Cai X.Y., PAn H., Wang Z.S., Geometric phases and quantum correlations of superconducting two-qubit system with dissipative effect, Physica C 520, 2016, pp. 8-18, DOI: 10.1016/j.physc.2015.11.004.

[41] Einstein A., Podolsky B., Rosen N., Can quantum-mechanical description of physical reality be considered complete?, Physical Review 47(10), 1935, pp. 777-780, DOI: 10.1103/PhysRev.47.777.

[42] Schrödinger E., Discussion of probability relations between separated systems, Mathematical Proceedings of the Cambridge Philosophical Society 31(4), 1935, pp. 555-565, DOI: $10.1017 /$ S0305004100013554; SCHRÖDINGER E., Probability relations between separated systems, Mathematical Proceedings of the Cambridge Philosophical Society 32(3), 1936, pp. 446-452, DOI: 10.1017/S0305004100019137.

[43] Bell J.S., On the Einstein Podolsky Rosen paradox, Physics Physique Fizika 1(3), 1964, pp. 195-200, DOI: 10.1103/PhysicsPhysiqueFizika.1.195.

[44] Adami C., Cerf N.J., von Neumann capacity of noisy quantum channels, Physical Review A 56(5), 1997, pp. 3470-3483, DOI: 10.1103/PhysRevA.56.3470.

[45] KÜBler O., Zeн H.D., Dynamics of quantum correlations, Annals of Physics 76(2), 1973, pp. 405-418, DOI: $10.1016 / 0003-4916(73) 90040-7$.

[46] Luo S., Quantum discord for two-qubit systems, Physical Review A 77(4), 2008, article 042303, DOI: 10.1103 /PhysRevA.77.042303.

[47] Ali M., Rau A.R.P., Alber G., Quantum discord for two-qubit X states, Physical Review A 81(4), 2010, article 042105, DOI: 10.1103/PhysRevA.81.042105. 